MPI H-V19-1997

DPNU-97-22

\title{
Zero Mode and Symmetry Breaking on the Light Front
}

\author{
Sho Tsujimaru ${ }^{a}$ 血 and Koichi Yamawaki ${ }^{b}$ ? \\ ${ }^{a}$ Max-Planck-Institut für Kernphysik, D-69029 Heidelberg \\ ${ }^{b}$ Department of Physics, Nagoya University, Nagoya 464-01, Japan
}

\begin{abstract}
We study the zero mode and the spontaneous symmetry breaking on the light front (LF). We use the discretized light-cone quantization (DLCQ) of Maskawa-Yamawaki to treat the zero mode in a clean separation from all other modes. It is then shown that the Nambu-Goldstone (NG) phase can be realized on the trivial LF vacuum only when an explicit symmetry-breaking mass of the NG boson $m_{\pi}$ is introduced. The NG-boson zero mode integrated over the LF must exhibit singular behavior $\sim 1 / m_{\pi}^{2}$ in the symmetric limit $m_{\pi} \rightarrow 0$, which implies that current conservation is violated at zero mode, or equivalently the LF charge is not conserved even in the symmetric limit. We demonstrate this peculiarity in a concrete model, the linear sigma model, where the role of zero-mode constraint is clarified. We further compare our result with the continuum theory. It is shown that in the continuum theory it is difficult to remove the zero mode which is not a single mode with measure zero but the accumulating point causing uncontrollable infrared singularity. A possible way out within the continuum theory is also suggested based on the " $\nu$ theory". We finally discuss another problem of the zero mode in the continuum theory, i.e., no-go theorem of Nakanishi-Yamawaki on the non-existence of LF quantum field theory within the framework of Wightman axioms, which remains to be a challenge for DLCQ, " $\nu$ theory" or any other framework of LF theory.
\end{abstract}

To appear in Physical Review D.

\footnotetext{
${ }^{1}$ E-mail address: sho@mickey.mpi-hd.mpg.de

${ }^{2}$ E-mail address: yamawaki@eken.phys.nagoya-u.ac.jp
} 


\section{Introduction}

Recent revival of the light-front (LF) quantization [1] aims at establishing a new formulation to study non-perturbative dynamics [2, 3]. A striking feature of the LF field theories is that the LF vacuum is simple, or even trivial [4]. The subtlety on this conclusion due to the so-called "zero mode" was first addressed back in 1976 by Maskawa and Yamawaki [5] who proposed the discretized light-cone quantization (DLCQ) P to treat the zero mode in a clean separation from other modes. They found a constraint equation for the zero mode ("zeromode constraint") through which the zero mode becomes dependent on other modes and can in principle be removed from the physical Fock space by solving the zero-mode constraint, thus establishing the trivial vacuum in $D L C Q$.

Based on the notion of this trivial vacuum, the first application of DLCQ to nonperturbative calculation was done in 1985 by Pauli and Brodsky [7] in the context of (1+1)dimensional Yukawa model: The DLCQ offers the promising prescription for obtaining the relativistic wave functions and the bound-state spectra in gauge theories. Their scheme has been applied to various models such as $\phi_{2}^{4}$ theory [8], Abelian [9] as well as non-Abelian [10] gauge theories in $(1+1)$ dimensions and the models in four dimensions [11]. As far as the two-dimensional models are concerned, reasonable correspondences with the known results have been obtained.

In spite of the success in two dimensions, there are a number of problems which must be solved to apply the same method to realistic models in four dimensions such as QCD. One of such problems is the long-standing zero-mode problem [5]. While the triviality of the LF vacuum in DLCQ can be achieved by solving out the zero mode from the physical Fock space through the zero-mode constraint [5], such a trivial vacuum would confront the

\footnotetext{
${ }^{3}$ The name "light-cone quantization" is actually confusing, since it is not on the light cone but on the light front which agrees with the former only in $1+1$ dimensions. However, here we simply follow the conventional naming of the majority of the literature. The DLCQ was also considered by Casher [6] independently in a different context.
} 
usual picture of the complicated non-perturbative vacuum structure in the conventional equal-time quantization such as the confinement and the spontaneous symmetry breaking (SSB). Simplicity of the LF vacuum and states can in fact only be realized at the sacrifice of simplicity of the operator side: The only operator responsible for such phenomena should be the zero mode whose constraint actually carries essential information of the complicated dynamics. One might thus expect that explicit solution of the zero-mode constraint in DLCQ should give rise to the physics equivalent to the nontrivial vacuum structure in equal-time quantization, while preserving the trivial LF vacuum. Actually, such an idea was carried out in the case of $(1+1)$-dimensional $\phi^{4}$ model [12, 13, 14] where it was argued that the solution of the zero-mode constraint might lead to the SSB (of a discrete symmetry). However, the most outstanding feature of the SSB is the existence of the Nambu-Goldstone (NG) boson associated with the continuous symmetry breaking in four dimensions.

In this paper, we elaborate on our previous paper [15] to examine how the NG boson in four dimensions can be described on the LF in view of the zero mode in DLCQ. The main conclusion of the previous work was that contrary to the naive expectation mentioned above, solving the zero-mode constraint does not lead to the NG phase, unless we introduce an explicit symmetry breaking (mass of the NG boson). The NG phase can only be realized when the NG-boson zero mode integrated over the LF behaves as $\sim 1 / m_{\pi}^{2}$ in the symmetry limit $m_{\pi}^{2} \rightarrow 0$. The most striking feature of its consequence is that the LF charge (zero mode of the local current) corresponding to the SSB is not conserved even in the symmetry limit $m_{\pi}^{2} \rightarrow 0$

Following Maskawa and Yamawaki [5] we formulate the canonical DLCQ á la Dirac for the scalar theory with a periodic boundary condition. Then the zero mode can be treated separately from other mode and be removed out of the physical Fock space through the zeromode constraint [5], thus leaving the LF vacuum trivial. Now that the vacuum is trivial, whole information about the SSB in the LF quantization should reside in the operator instead of the state, namely, in the zero mode whose dynamics is governed by the zero-mode 
constraint.

However, direct application of the zero-mode constraint leads to inconsistent result: The DLCQ allows neither the emission vertex of the NG boson nor the corresponding current vertex, as far as the NG boson mass is exactly zero, or conservation of the LF charge is imposed ("(false) no-go theorem") [15]. Namely, solving the zero-mode constraint does not give rise to $S S B$ at all in the exact symmetric case $m_{\pi}^{2} \equiv 0$, in contradiction to the naive expectation mentioned above [12, 13, 14].

In order to recover the NG phase in DLCQ with the trivial vacuum, we thus need to formulate non-conservation of the LF charge. We propose [15] that it can be achieved in DLCQ by first introducing explicit-symmetry-breaking mass of the NG boson $m_{\pi}$ and then taking its massless limit. This mass plays a role of regularization of the infrared singularity of the zero mode. Based on the notion of PCAC, it will be clarified how the SSB without NG-boson mass becomes self-contradictory in DLCQ and how the arguments leading to the above "(false) no-go theorem" went wrong. We find that the NG phase on the LF is characterized by the singular behavior of the NG-boson zero mode: The global zero mode (zero mode integrated over the LF) of the NG boson must be proportional to $1 / m_{\pi}^{2}$ in the symmetric limit $m_{\pi} \rightarrow 0$ [15]. This in fact leads to non-conservation of the LF charge while preserving the trivial vacuum.

The above general feature of the SSB on the LF will be further demonstrated in a concrete field theoretical model, the linear sigma model, in which the role of the zero mode is most explicitly illustrated. We derive coupled zero-mode constraints with the NG-boson mass included and solve them in perturbation around the classical broken solution which corresponds to the classical broken vacuum in the equal-time quantization. The singular behavior of the NG-boson zero mode is indeed explicitly demonstrated by such a perturbative solution which at tree level yields non-vanishing $\sigma \pi \pi$ and $\mathrm{NN} \pi$ vertices consistently with the usual result of the equal-time quantization. It is most remarkable that the current conservation or the LF-charge conservation actually breaks down due to such a singular 
behavior of the NG boson zero mode.

We also note that were it not for the NG boson mass from the onset, the zero-mode constraints, after integration over the transverse coordinate, would take an essentially the same form as that of the two-dimensional massless scalar theory which, however, will be shown to be ill-defined in accord with Coleman's theorem [16]. Thus the LF theory without NG-boson mass in four dimensions is also ill-defined and hence introduction of the NG-boson mass and the resulting non-conservation of the LF charge is inevitable in DLCQ.

Finally, we shall compare our result with the zero mode problem in the continuum theory. In the continuum theory it is rather difficult to remove the zero mode in a sensible manner as was pointed out by Nakanishi and Yamawaki [17] long time ago: The real problem is not a single mode with $p^{+} \equiv 0$ (which is merely of zero measure and harmless) but actually the accumulating point $p^{+} \rightarrow 0$ as can been seen from $1 / p^{+}$singularity in the Fourier transform of the sign function $\epsilon\left(x^{-}\right)$appearing in the canonical commutator on LF. This prevents us from constructing even a free theory on the LF (no-go theorem [17]), which actually cannot be overcome either by taking the simple continuum limit $L \rightarrow \infty$ of DLCQ nor any other existing method [17]. Besides this most difficult problem, in this paper we shall point out another problem in the continuum theory. Namely, the SSB charge on LF does not annihilate the vacuum, if we formulate the sigma model with SSB on the LF with careful treatment of the boundary condition. Even if we pretend to have removed the zero mode, as far as the canonical commutator takes the form of the sign function $\epsilon\left(x^{-}\right)$, it inevitably leads to a nontrivial vacuum, namely, the LF charge does not annihilate the vacuum. This in fact corresponds to difficulty to remove the zero mode as the accumulating point mentioned above (in contradiction to a widely spread expectation [3]).

We then suggest that a possible way out of this problem within the continuum theory would be the " $\nu$-theory" proposed by Nakanishi and Yamawaki [17] which removes the zero mode in the continuum theory by shaving the vicinity of the zero mode in such a way that the sign function in the commutator is modified to a certain function vanishing at $x^{-}= \pm \infty$. 
This theory is expected to yield the same result as that we obtain in this paper based on DLCQ, although it does not overcome the no-go theorem [17] mentioned above. The no-go theorem and the $\nu$-theory will be further discussed in great detail in order to remind the reader of the old results [17].

The plan of this paper is as follows: In Sec.2 we recapitulate the canonical formalism of DLCQ for the scalar theory and the zero-mode constraint in a way slightly different from the original one [5]. In Sec.3 we consider the SSB of the continuous symmetry and show how the NG phase can be realized in the trivial LF vacuum through the explicit-symmetry-breaking mass of the NG boson. The singular behavior of the global zero mode of the NG boson in the $m_{\pi} \rightarrow 0$ is required, which implies non-conservation of the SSB current. In Sec.4 we apply our formulation to the linear sigma model by treating the zero-mode constraints explicitly. It is shown that the tree-level amplitude of both the $\sigma \pi \pi$ and the $N N \pi$ scatterings are actually obtained in DLCQ due to the singular behavior of the NG-boson zero mode in the symmetric limit. In Sec.5 we discuss the zero-mode problem in the continuum theory, which is quite different from that in DLCQ. Sec.6 is devoted to the Summary and Discussions where we present particular discussions on the no-go theorem and the $\nu$-theory in detail. A detailed derivation of the DLCQ canonical commutator is given in Appendix A. In Appendix B the special status of the boundary condition on the LF is discussed. In Appendix C, we describe the unbroken phase of $O(2)$-symmetric linear sigma model in DLCQ and check the operator ordering (Weyl ordering) we use in discussing the perturbative solution. The property of the higher order perturbative solutions of the zero-mode constraints is studied in Appendix D.

\section{Zero Mode in the Discretized Light-Cone Quantiza- tion}

In this section we review for later purpose the canonical DLCQ of the scalar theory [5]. Throughout this paper we use the convention of the LF coordinate $x^{\mu}=\left(x^{+}, \vec{x}\right)=\left(x^{+}, x^{\perp}, x^{-}\right)$, 
where

$$
\begin{aligned}
& x^{ \pm} \equiv \frac{1}{\sqrt{2}}\left(x^{0} \pm x^{3}\right) \\
& x^{\perp} \equiv\left(x^{1}, x^{2}\right)
\end{aligned}
$$

The quantization surface on the equal "LF time" $x^{+}$is defined in the finite region $-L \leq x^{-} \leq$ $L$ [5, 6, 7], while no such restriction is necessarily imposed for the transverse coordinates $x^{\perp}$. The "continuum" limit $L \rightarrow \infty$ (or, more precisely, infinite volume limit) is taken at the final stage of the whole calculations. We use the notation $\int d^{3} \vec{x} \equiv \lim _{L \rightarrow \infty} \int_{-L}^{L} d x^{-} d^{2} x^{\perp}$.

Let us consider the self-interacting scalar theory in four dimensions whose Lagrangian is expressed in terms of the LF coordinate as

$$
\mathcal{L}=\partial_{+} \phi \partial_{-} \phi-\frac{1}{2}\left(\partial_{\perp} \phi\right)^{2}-\frac{1}{2} \mu^{2} \phi^{2}-V(\phi)
$$

where $V(\phi)$ is a potential. The canonical momentum conjugate to $\phi(x)$ is

$$
\pi(x)=\frac{\partial \mathcal{L}}{\partial\left(\partial_{+} \phi\right)}=\partial_{-} \phi(x)
$$

which leads to a primary constraint of the theory:

$$
\Phi(x)=\pi(x)-\partial_{-} \phi(x) \approx 0
$$

Since $x^{-}$is restricted to the finite region, the boundary condition should be specified at $x^{-}= \pm L$. We adopt the periodic boundary condition on $x^{-}$[5], which is consistent with non-vanishing vacuum expectation value of the scalar field. In fact, very existence of the zero mode is related to this periodic boundary condition. Other boundary conditions such as the anti-periodic one will be discussed in Appendix B. Owing to the boundary condition in the finite box, all surface terms can be treated unambiguously, while their treatment is subtle in the continuum framework (see Sect. 5 and Appendix B). Actually, as was emphasized by Steinhardt [18], the boundary condition should always be specified even in the "continuum " theory in order to have a consistent LF quantization. In fact, the boundary condition on 
LF includes a part of the dynamics in sharp contrast to the equal-time quantization. That is, different boundary condition defines a different theory. We shall clarify a special role of the boundary condition on the LF in Appendix B.

Since the zero mode in DLCQ is clearly separated from other modes, we may make an orthogonal decomposition of the primary constraint into two parts as follows [19]. Let us divide the scalar field $\phi(x)$ into the oscillating modes $\varphi(x)$ plus the zero mode $\phi_{0}\left(x^{+}, x^{\perp}\right)$ :

$$
\begin{aligned}
\phi(x) & =\varphi(x)+\phi_{0}\left(x^{+}, x^{\perp}\right), \\
\phi_{0} & \equiv \frac{1}{2 L} \int_{-L}^{L} \phi(x) d x^{-} .
\end{aligned}
$$

The conjugate momentum $\pi$ may also be divided as

$$
\pi(x)=\pi_{\varphi}(x)+\pi_{0}\left(x^{+}, x^{\perp}\right)
$$

where $\pi_{0}$ and $\pi_{\varphi}$ are the zero modes conjugate to $\phi_{0}$ and that to the remaining orthogonal part $\varphi(x)$, respectively. Now, substituting (2.6) and (2.8) into (2.5), we have two independent constraints,

$$
\Phi_{1}(x) \equiv \pi_{\varphi}(x)-\partial_{-} \varphi(x) \approx 0
$$

and

$$
\Phi_{2}(x) \equiv \pi_{0}\left(x^{+}, x^{\perp}\right) \approx 0
$$

in place of the original one (2.5).

From the fundamental Poisson bracket

$$
\{\phi(x), \pi(y)\}=\delta^{(3)}(\vec{x}-\vec{y})
$$

we obtain

$$
\left\{\phi_{0}, \pi_{0}\right\}=\frac{1}{2 L} \delta^{(2)}\left(x^{\perp}-y^{\perp}\right), \quad\left\{\phi_{0}, \phi_{0}\right\}=\left\{\pi_{0}, \pi_{0}\right\}=0
$$

and

$$
\left\{\varphi(x), \pi_{\varphi}(y)\right\}=\left\{\delta\left(x^{-}-y^{-}\right)-\frac{1}{2 L}\right\} \delta^{(2)}\left(x^{\perp}-y^{\perp}\right)
$$




$$
\{\varphi(x), \varphi(y)\}=\left\{\pi_{\varphi}(x), \pi_{\varphi}(y)\right\}=0
$$

where $x^{+}=y^{+}$is understood. All other Poisson brackets are equal to zero as expected.

The total Hamiltonian is obtained by adding the primary constraints to the canonical one $H_{c}$ :

$$
\begin{aligned}
H_{T} & \equiv H_{c}+\int d^{3} \vec{x}\left[v_{1}(x) \Phi_{1}(x)+v_{2}(x) \Phi_{2}(x)\right], \\
H_{c} & =\int d^{3} \vec{x}\left[\frac{1}{2}\left\{\left(\partial_{\perp} \phi\right)^{2}+\mu^{2} \phi^{2}\right\}+V(\phi)\right],
\end{aligned}
$$

where $v_{2}$ and $v_{1}$ are the zero mode and the remaining part of the Lagrange multiplier, respectively. The multiplier $v_{1}$ is determined by the consistency condition for $\Phi_{1}(x)$ through the relation

$$
\partial_{-} v_{1}(x)=\frac{1}{2}\left\{\pi(x), H_{c}\right\}-\frac{1}{2}\left\{\pi_{0}, H_{c}\right\},
$$

which can be easily integrated without ambiguity owing to the periodic boundary condition. On the other hand, the consistency condition for $\Phi_{2}(x)$

$$
\dot{\Phi}_{2}(x)=\left\{\Phi_{2}(x), H_{T}\right\}=\frac{1}{2 L} \int_{-L}^{L} d x^{-}\left[\left(\partial_{\perp}^{2}-\mu^{2}\right) \phi-\frac{\partial V}{\partial \phi}\right] \approx 0
$$

leads to a new constraint so-called "zero-mode constraint" [5]:

$$
\Phi_{3}(x) \equiv \frac{1}{2 L} \int_{-L}^{L} d x^{-}\left[\left(\mu^{2}-\partial_{\perp}^{2}\right) \phi+\frac{\partial V}{\partial \phi}\right] .
$$

The consistency condition for the zero-mode constraint yields no further constraint and just determines the multiplier $v_{2}$. Note that in deriving these relations we have used the condition

$$
\delta\left(x^{-}-L\right)=\delta\left(x^{-}+L\right)
$$

which comes from the definition of the delta function with the periodic boundary condition:

$$
\delta\left(x^{-}\right)=\frac{1}{2 L} \sum_{n \in \mathbf{Z}} e^{\frac{i n \pi}{L} x^{-}}
$$


Having obtained all the second-class constraints, we are ready to calculate the Dirac bracket of two arbitrary dynamical variables $A(x)$ and $B(y)$ as

$$
\begin{aligned}
\{A(x), B(y)\}_{D B} & \equiv\{A(x), B(y)\} \\
& -\sum_{i, j} \int d^{3} \vec{u} \int d^{3} \vec{v}\left\{A(x), \Phi_{i}(u)\right\}\left(C^{-1}\right)_{i, j}(u, v)\left\{\Phi_{j}(v), B(y)\right\}
\end{aligned}
$$

where $\left(C^{-1}\right)_{i, j}$ is the inverse of $C_{i, j}(x, y) \equiv\left\{\Phi_{i}(x), \Phi_{j}(y)\right\}$ which is the matrix of Poisson bracket of the constraints. The inverse matrix can be calculated by noting the separation of the zero mode from other modes. For instance, the matrix element

$$
\begin{aligned}
C_{1,1}(x, y) \equiv\left\{\Phi_{1}(x), \Phi_{1}(y)\right\} & =\left(\partial_{-}^{y}-\partial_{-}^{x}\right) \delta\left(x^{-}-y^{-}\right) \cdot \delta^{(2)}\left(x^{\perp}-y^{\perp}\right) \\
& =\frac{1}{2 L} \sum_{n \in \mathbf{Z}}\left(\frac{-2 i n \pi}{L}\right) e^{\frac{i n \pi}{L}\left(x^{-}-y^{-}\right)} \cdot \delta^{(2)}\left(x^{\perp}-y^{\perp}\right)
\end{aligned}
$$

has its inverse

$$
\begin{aligned}
{\left[C_{1,1}(x, y)\right]^{-1} } & =-\frac{1}{4}\left\{\epsilon\left(x^{-}-y^{-}\right)-\frac{x^{-}-y^{-}}{L}\right\} \cdot \delta^{(2)}\left(x^{\perp}-y^{\perp}\right) \\
& =\frac{1}{2 L} \sum_{n \neq 0}\left(\frac{-L}{2 i n \pi}\right) e^{\frac{i n \pi}{L}\left(x^{-}-y^{-}\right)} \cdot \delta^{(2)}\left(x^{\perp}-y^{\perp}\right)
\end{aligned}
$$

in the sense that

$$
\int_{-L}^{L} d z^{-} \int d z^{\perp} C_{1,1}(x, z)\left[C_{1,1}(z, y)\right]^{-1}=\frac{1}{2 L} \sum_{n \neq 0, n \in \mathbf{Z}} e^{i \frac{n \pi}{L}\left(x^{-}-y^{-}\right)} \cdot \delta^{(2)}\left(x^{\perp}-y^{\perp}\right)
$$

where $\epsilon(x)$ is the sign function satisfying $\partial_{x} \epsilon(x)=2 \delta(x)$. Note that the right hand side (r.h.s.) in (2.25) is a delta function minus zero-mode contribution as it should, since the zero mode is already subtracted from $\Phi_{1}$ beforehand.

After the Dirac bracket is taken, all the second-class constraints become strong relations and so is the zero-mode constraint (2.19):

$$
\frac{1}{2 L} \int_{-L}^{L} d x^{-}\left[\left(\mu^{2}-\partial_{\perp}^{2}\right) \phi+\frac{\partial V}{\partial \phi}\right]=0
$$


which is further converted into the operator relation, when we pass to the quantum theory via the correspondence principle between the Dirac bracket and the commutator, \{\}$_{D B} \rightarrow$ $-i[$ ]. This implies that the zero mode is not an independent degree of freedom but is implicitly written in terms of other oscillating modes. It was actually the central issue of Maskawa and Yamawaki [5] who claimed that such a constrained zero mode can in principle be solved away out of the physical Fock space and hence the trivial LF vacuum is justified in $D L C Q$. It is also noted [20] that the zero-mode constraint (2.26) can also be obtained by simply integrating in $x^{-}$the Euler-Lagrange equation $\left(\square+\mu^{2}\right) \phi=-\frac{\partial V}{\partial \phi}$ with use of the periodic boundary condition:

$$
0=-\int_{-L}^{L} d x^{-} 2 \partial_{+} \partial_{-} \phi=\int_{-L}^{L} d x^{-}\left[\left(\mu^{2}-\partial_{\perp}^{2}\right) \phi+\frac{\partial V}{\partial \phi}\right] .
$$

Namely, the zero mode constraint is a part of the equation of motion and the zero mode is nothing but an auxiliary field having no kinetic term.

Through the above correspondence principle, we obtain from (2.22) the canonical commutation relation [0]:

$$
[\varphi(x), \varphi(y)]=-\frac{i}{4}\left\{\epsilon\left(x^{-}-y^{-}\right)-\frac{x^{-}-y^{-}}{L}\right\} \delta^{(2)}\left(x^{\perp}-y^{\perp}\right)
$$

for the field without zero mode, which is a direct consequence of (2.24). In sharp contrast to the sign function in the continuum theory (see Sect.5), here in DLCQ we observe presence of the extra term $\left(x^{-}-y^{-}\right) / L$ in the commutator (2.28), which is nothing but a term subtracting the zero mode as can be seen from (2.24). Note that (2.28) is the same as the commutation relation of the full field $\phi$ in the free theory in which the zero mode becomes identically zero through the zero-mode constraint (2.26).

By computing (2.22) for the full matrix, we further obtain a commutation relation for the full field including the zero mode (see Appendix A):

$$
\begin{aligned}
{[\phi(x), \phi(y)]=} & -\frac{i}{4} \int d u^{-} d v^{-}\left[\beta\left(x^{\perp}\right) \alpha\left(u^{-}, x^{\perp}\right)-\delta\left(u^{-}-x^{-}\right)\right]\left\{\epsilon\left(u^{-}-v^{-}\right)-\frac{u^{-}-v^{-}}{L}\right\} \times \\
& {\left[\beta\left(y^{\perp}\right) \alpha\left(v^{-}, y^{\perp}\right)-\delta\left(v^{-}-y^{-}\right)\right] \delta^{(2)}\left(x^{\perp}-y^{\perp}\right), }
\end{aligned}
$$


where

$$
\alpha(\vec{x}) \equiv \mu^{2}-\partial_{\perp}^{2}+\frac{\partial^{2} V}{\partial \phi^{2}} \quad, \quad \beta^{-1}\left(x^{\perp}\right) \equiv \int_{-L}^{L} d x^{-} \alpha(\vec{x})
$$

At first sight, (2.29) looks different from the original expression in [5]:

$$
[\phi(x), \phi(y)]=-\frac{i}{4}\left\{\epsilon\left(x^{-}-y^{-}\right)-2 \beta \int_{y^{-}}^{x^{-}} \alpha\left(z^{-}\right) d z^{-}\right\} \delta^{(2)}\left(x^{\perp}-y^{\perp}\right)
$$

which was obtained without orthogonal decomposition of the primary constraint (2.5) into two parts, zero mode and non-zero modes. However, explicit computation shows (Appendix A) that both are in fact equivalent to each other if the operator ordering is disregarded (i.e., in the sense of Dirac brackets). Note that $\alpha$ and $\beta$ contain field operators in the interacting theory and hence (2.29) (or (2.31)) is generally a complicated operator-valued commutation relation, which is nothing but a consequence of the constrained zero mode carrying the vital dynamical information of the theory.

Here we should remark on the operator ordering to be consistent with the Dirac quantization. In the Dirac procedure, the Dirac bracket is constructed so that all the second class constraints can automatically hold as strong identities. This property must be preserved in passing from the classical theory to the quantum one. In the case at hand, the zero-mode constraint should commute with any operator just by calculation using the commutator for the full scalar fields. Rather such operator orderings in the quantum theory must be determined for both the zero-mode constraint and the r.h.s. of (2.29) (or (2.31)) simultaneously. However this is an extremely difficult task and we take a different approach: Instead of a requirement for the zero-mode constraint to be a strong operator identity, we assume the Weyl ordering for the operators in the zero-mode constraint to solve it explicitly. The solution of the zero mode then leads to the commutator (2.29) with a definite operator ordering. Our choice of the Weyl ordering is based on the general argument [21]. Moreover it will be justified through the study of linear sigma model in the section 4 and Appendix C. 


\section{Nambu-Goldstone Boson on the Light Front}

It is now widely believed that non-perturbative phenomena due to nontrivial vacuum in equal-time quantization can be understood in the LF quantization through the operator property of the zero mode. Among the various zero modes, we focus on the bosonic zero mode which has been shown to be a dependent degree of freedom and is expected to play a key role to realize SSB on the LF. One might then expect that the non-perturbative vacuum structure in equal-time quantization is simply replaced by the solution of the zero-mode constraint. However the problem is not so simple for the realistic case with continuous symmetry in four dimensions, whose realization is usually associated with the massless NG boson.

The purpose of this section is to propose the criteria for global continuous symmetry breaking on the trivial LF vacuum [15]. Before drawing our main conclusion, we first show

that the naive application of the zero-mode constraint will not lead to the NG phase at all in contradiction to the above expectation (" false) no-go theorem" [15]): If the zero-mode constraint is not reguralized by the explicit mass of the $N G$ boson, the NG phase cannot be realized in DLCQ. Namely, there is no coupling of NG boson as well as no associated current vertex at $q^{2}=0$, where $q^{\mu}$ is the momentum of the $N G$ boson.

In order to confirm our assertion, let us start with assuming that the NG phase is already realized on the LF in the presence of an exactly conserved current and examine its consequence. Consider the arbitrary NG-boson emission process $A \rightarrow B+\pi$, where both A and $B$ represent one-particle states which couple with the NG boson. The index of the NG boson associated with the internal symmetry is omitted for simplicity.

Based on the reduction formula, the transition amplitude may be written as

$$
\begin{aligned}
& \langle B, \pi(q) \mid A\rangle \equiv i \int d^{4} x e^{i q x}\langle B|\square \pi(x)| A\rangle \\
& \quad=i(2 \pi)^{4} \delta\left(p_{A}^{-}-p_{B}^{-}-q^{-}\right) \delta^{(3)}\left(\vec{p}_{A}-\vec{p}_{B}-\vec{q}\right)\left\langle B\left|j_{\pi}(0)\right| A\right\rangle
\end{aligned}
$$


where $\pi(x)$ and $j_{\pi}(x)=\square \pi(x)=\left(2 \partial_{+} \partial_{-}-\partial_{\perp}^{2}\right) \pi(x)$ are the interpolating field of NG boson, which is exactly massless, and the source function of the NG boson, respectively, and $q^{\mu}=p_{A}^{\mu}-p_{B}^{\mu}$ is the NG-boson four momenta.

Taking the collinear momentum frame [22], $q^{+}=q^{\perp}=0$ and $q^{-} \neq 0$ which is not soft momentum for the on-shell NG boson with $q^{2}=0$, we find that the NG-boson emission vertex does vanish as follows:

$$
\begin{aligned}
& (2 \pi)^{3} \delta^{(3)}\left(\vec{p}_{A}-\vec{p}_{B}\right)\left\langle B\left|j_{\pi}(0)\right| A\right\rangle \\
& \quad=\int d^{3} \vec{x}\left\langle B\left|\left(2 \partial_{+} \partial_{-}-\partial_{\perp}^{2}\right) \pi\right| A\right\rangle \\
& \quad=\int d^{2} x^{\perp} \lim _{L \rightarrow \infty}\left\langle B\left|\left(\int_{-L}^{L} d x^{-} 2 \partial_{+} \partial_{-} \pi\right)\right| A\right\rangle=0,
\end{aligned}
$$

where the periodic boundary condition was used for the NG boson field $\pi$ as before. As seen from (2.27), the last line is nothing but a zero-mode constraint for the massless field, and hence the zero-mode constraint itself dictates that the NG boson vertex should vanish. Thus we have established that the solution of the zero-mode constraint, whether perturbative or non-perturbative or even exact, does not lead to the NG phase at all.

Another symptom of this disease is the vanishing of the current vertex for the SSB current as a direct consequence of the LF charge conservation, which again comes from our periodic boundary condition through the local current conservation. The current vertex is an analogue of $g_{A}$ for the nucleon matrix element and is related to the NG boson vertex $\left(g_{N N \pi}\right.$ for the nucleon case) in the usual SSB argument through the analogue of the Goldberger-Treiman relation. (Caveat for the nucleon case will be given later.) Now that we have seen that the NG boson vertex vanishes due to the periodic boundary condition, we may naturally guess that the current vertex also should for the same reason. In what follows we shall argue that this is indeed the case. In the NG phase the current $J_{\mu}$ is divided into the pole term consisting of an interpolating field of the NG boson and the remaining non-pole term, that is,

$$
J_{\mu}=-f_{\pi} \partial_{\mu} \pi+\widehat{J}_{\mu}
$$


where $f_{\pi}$ is the "decay constant" of the NG boson and $\widehat{J}_{\mu}$ denotes the non-pole term which yields the current vertex. Now, integrating the local current conservation over the LF, we find that the NG-boson pole term drops out, leaving only the non-pole term due to the periodic boundary condition as before. Then we establish the vanishing current vertex as follows [15]:

$$
\begin{aligned}
0 & =\left\langle B\left|\int d^{3} \vec{x} \partial_{\mu} \widehat{J}^{\mu}(x)\right| A\right\rangle_{x^{+}=0} \\
& =-i(2 \pi)^{3} \delta^{(3)}(\vec{q}) \frac{m_{A}^{2}-m_{B}^{2}}{2 p_{A}^{+}}\left\langle B\left|\widehat{J}^{+}(0)\right| A\right\rangle,
\end{aligned}
$$

where $q^{-}=p_{A}^{-}-p_{B}^{-}=\left(m_{A}^{2}-m_{B}^{2}\right) / 2 p_{A}^{+}$, which implies

$$
\left\langle B\left|\widehat{J}^{+}(0)\right| A\right\rangle=0
$$

as far as $m_{A}^{2} \neq m_{B}^{2}$. The current vertex $\left\langle B\left|\widehat{J}^{+}(0)\right| A\right\rangle$ at $q^{2}=0$ is nothing but the matrix element of LF charge $\widehat{Q} \equiv \int d^{3} \vec{x} \widehat{J}^{+}$constructed only from the non-pole term (well-defined charge even in the SSB phase) and is essentially the same as " $X$ matrix" of Weinberg [22]. The chiral algebra of LF charge actually yields the celebrated Adler-Weisberger sum rule [24] and its extensions as an algebraic realization of the chiral symmetry in terms of the notion of representation mixings among hadronic states with non-degenerate masses $m_{A}^{2} \neq m_{B}^{2}$ 22, 25, 26]. Hence the vanishing of the current vertex invalidates whole success of the Adler-Weisberger sum rules and the associated representation mixings. Actually, vanishing of the current vertex means conservation of the LF charge $\widehat{Q}$ which immediately follows from conservation of the full LF charge $Q \equiv \int d^{3} \vec{x} J^{+}$, since $Q$ always reduces to $\widehat{Q}$, with the pole part being dropped out of $Q$ due to the integration over the LF:

$$
\left[\widehat{Q}, P^{-}\right]=\left[Q, P^{-}\right]=0
$$

\footnotetext{
${ }^{4}$ For the case where the two particles $\mathrm{A}$ and $\mathrm{B}$ have a degenerate mass, i.e. $m_{A}=m_{B}$, Eq. (3.4) by itself does not implies the vanishing current vertex $\left\langle B\left|\widehat{J}^{+}(0)\right| A\right\rangle=0$. However, in this case $q^{2} \rightarrow 0$ corresponds to the soft momentum limit $q^{\mu} \rightarrow 0$, which implies that even in the usual equal-time treatment, the NG-boson emission vertex vanishes anyway by the low energy theorem, even when the current vertex is non-zero (for the nucleon case, the current vertex is also zero for kinematical reason, although $g_{A}$ is non-zero, see the discussion in Sect.4) 23.
} 
We again emphasize that conservation of the LF charges is a direct consequence of the periodic boundary condition we are using, provided that the local current is conserved as we imposed.

So, what went wrong? One might use other boundary conditions than the periodic one. In Appendix B we shall argue that beside the periodic boundary condition, only the antiperiodic one can be consistent in DLCQ, which however yields no SSB because of obvious absence of the zero mode. One might then give up DLCQ and consider the continuum theory from the onset, in which case, however, we still need to specify the boundary condition in order to have a consistent LF theory [18] as we shall discuss also in Appendix B. The best we can do in the continuum theory will be described in Sect. 5, which, although can give non-zero NG boson vertex and current vertex (i.e., non-conservation of the LF charge) due to the boundary condition, will result in another disaster, namely, the LF charge does not annihilate the vacuum, thus invalidating the trivial vacuum as the greatest advantage of the whole LF approach. One also might suspect that the finite volume in $x^{-}$direction in DLCQ could be the cause of this NG-boson decoupling, since it is well known that SSB does not occur in the finite volume. However, we actually take the $L \rightarrow \infty$ limit in the end, and such a limit in fact must realize SSB as was demonstrated in the equal-time quantization in the infinite volume limit of the finite box quantization [27]. Moreover, in the case at hand in four dimensions, the transverse directions $x^{\perp}$ extend to infinity. Hence this argument is totally irrelevant any way.

Therefore the above result is not an artifact of the periodic boundary condition and DLCQ but is deeply connected to the very nature of the LF quantization, namely the zero mode. Thus, as far as the trivial property of the LF vacuum is to be maintained, the only way to recover the NG phase seems to break the symmetry explicitly. By the various arguments to follow along this line both in this section and Sect.4, we actually conclude [15]:

The NG phase can be realized in DLCQ only when the $N G$-boson mass $m_{\pi}$ is introduced into the theory. The non-vanishing NG-boson emission vertex as well as the corresponding 
current vertex at $q^{2}=0$ is recovered through the explicit symmetry-breaking term in the $m_{\pi} \rightarrow 0$ limit, which is characterized by the singular behavior of the NG-boson zero mode $\omega_{\pi}:$

$$
\int d^{3} \vec{x} \omega_{\pi} \sim \frac{1}{m_{\pi}^{2}}
$$

We can easily confirm (3.7) with the help of the PCAC hypothesis: $\partial_{\mu} J^{\mu}(x)=f_{\pi} m_{\pi}^{2} \pi(x)$. Since the current divergence of the non-pole term $\widehat{J}^{\mu}(x)$ reads $\partial_{\mu} \widehat{J}^{\mu}(x)=f_{\pi}\left(\square+m_{\pi}^{2}\right) \pi(x)=$ $f_{\pi} j_{\pi}(x)$, we obtain

$$
\begin{aligned}
\left\langle B\left|\int d^{3} \vec{x} \partial_{\mu} \widehat{J}^{\mu}(x)\right| A\right\rangle & =f_{\pi} m_{\pi}^{2}\left\langle B\left|\int d^{3} \vec{x} \pi(x)\right| A\right\rangle \\
& =\left\langle B\left|\int d^{3} \vec{x} f_{\pi} j_{\pi}(x)\right| A\right\rangle
\end{aligned}
$$

where the integration of the pole term $\square \pi(x)$ is dropped out as before. On the r.h.s. of (3.8), one can replace $\pi(x)$ by the zero mode $\omega_{\pi} \equiv \frac{1}{2 L} \int_{-L}^{L} d x^{-} \pi(x)$ because the oscillating modes drop out due to the periodic boundary condition. Suppose that $\int d^{3} \vec{x} \omega_{\pi}(x)=\int d^{3} \vec{x} \pi(x)$ is regular when $m_{\pi}^{2} \rightarrow 0$, this does not lead to the NG phase at all, because the remaining two terms then become vanishing. In order to have the non-zero NG-boson emission vertex (3.9) as well as the non-zero current vertex (1.h.s. of (3.8)) at $q^{2}=0$, the zero mode $\omega_{\pi}$ must behave as (3.7).

This implies that at the quantum level the LF charge $Q=\widehat{Q}$ is not conserved or the current conservation does not hold for its particular Fourier component with $\vec{q}=0$ even in the symmetric limit:

$$
\dot{Q}=\frac{1}{i}\left[Q, P^{-}\right]=\left.\partial^{\mu} J_{\mu}\right|_{\vec{q}=0}=f_{\pi} \lim _{m_{\pi}^{2} \rightarrow 0} m_{\pi}^{2} \int d^{3} \vec{x} \omega_{\pi} \neq 0
$$

although we can recover the conserved current at the classical level.

The situation may well be clarified when we consider the general expression for the current 
matrix element in momentum space with an explicit symmetry breaking:

$$
\frac{m_{\pi}^{2} f_{\pi} j_{\pi}\left(q^{2}\right)}{m_{\pi}^{2}-q^{2}}=\partial^{\mu} J_{\mu}(q)=\frac{q^{2} f_{\pi} j_{\pi}\left(q^{2}\right)}{m_{\pi}^{2}-q^{2}}+\partial^{\mu} \widehat{J}_{\mu}(q)
$$

which is a weaker condition than the operator relation of PCAC hypothesis. What we have done in proving the absence of NG phase for the exactly conserved current ("(false) no-go theorem") is summarized as follows: We first set the 1.h.s. of (3.11) to zero or equivalently, assumed implicitly the regular behavior of $\int d^{3} \vec{x} \omega_{\pi}(x)$ in the massless limit in accord with the current conservation $\partial^{\mu} J_{\mu}=0$. Second, the first term (NG-boson pole term) on the r.h.s. of (3.11) vanishes rigorously due to the periodic boundary condition or the zero-mode constraint in the DLCQ with $\vec{q}=0\left(q^{2}=0\right)$. Thus we arrived at $\partial^{\mu} \widehat{J}_{\mu}(q)=0$ in addition to the vanishing of the NG-boson vertex. However, this procedure is equivalent to claiming the nonsense relation

$$
1=\lim _{m_{\pi}^{2}, q^{2} \rightarrow 0}\left(\frac{m_{\pi}^{2}-q^{2}}{m_{\pi}^{2}-q^{2}}\right)=0,
$$

as far as $f_{\pi} j_{\pi} \neq 0$ (NG phase). Therefore the " $m_{\pi}^{2} \equiv 0$ " theory with vanishing l.h.s. is ill-defined in DLCQ and we should define the symmetric limit after introducing the explicit symmetry-breaking term.

\section{The Sigma Model}

Based on DLCQ discussed in Sect.2, let us now demonstrate (3.7) by explicitly solving the zero-mode constraints with the NG-boson mass in a concrete model theory [15]. As the simplest but a nontrivial example, we consider $O(2)$-symmetric linear sigma model defined by the Lagrangian:

$$
\begin{aligned}
\mathcal{L}= & \bar{\psi} i \gamma^{\mu} \partial_{\mu} \psi-g \bar{\psi}\left(\sigma+i \gamma_{5} \pi\right) \psi+\frac{1}{2}\left(\partial_{\mu} \sigma\right)^{2}+\frac{1}{2}\left(\partial_{\mu} \pi\right)^{2} \\
& -\frac{1}{2} \mu^{2}\left(\sigma^{2}+\pi^{2}\right)-\frac{\lambda}{4}\left(\sigma^{2}+\pi^{2}\right)^{2}+c \sigma
\end{aligned}
$$

where $\mu^{2}<0, c$ is the symmetry-breaking parameter and $\psi$ is the "nucleon" $(N)$ field. We take $c \rightarrow 0$ at the final stage. 
In equal-time quantization the NG phase is well described even at the tree-level. It is then sufficient to demonstrate, by solving the constraints, that such a situation is realized also on LF. Two kinds of vertices will be examined below: the $\sigma \pi \pi$ vertex and the $N N \pi$ vertex.

\section{1 $\sigma \pi \pi$ vertex}

In this case it is adequate to restrict ourselves to the bosonic sector. The relevant Lagrangian is

$$
\mathcal{L}=\frac{1}{2}\left(\partial_{\mu} \sigma\right)^{2}+\frac{1}{2}\left(\partial_{\mu} \pi\right)^{2}-\frac{1}{2} \mu^{2}\left(\sigma^{2}+\pi^{2}\right)-\frac{\lambda}{4}\left(\sigma^{2}+\pi^{2}\right)^{2}+c \sigma
$$

As in Sect.2, we adopt the periodic boundary condition in DLCQ in order to allow the nonvanishing vacuum expectation value. The quantization can be done in the way similar to that in the one-component case given in Sect.2. There are two kinds of zero modes $\pi_{0} \equiv$ $\frac{1}{2 L} \int_{-L}^{L} d x^{-} \pi(x)$ and $\sigma_{0} \equiv \frac{1}{2 L} \int_{-L}^{L} d x^{-} \sigma(x)$ which are separated clearly from other oscillating modes, $\varphi_{\pi} \equiv \pi-\pi_{0}$ and $\varphi_{\sigma} \equiv \sigma-\sigma_{0}$, respectively. The canonical commutation relation for the oscillating modes (2.28) now reads:

$$
\left[\varphi_{i}(x), \varphi_{j}(y)\right]=-\frac{i}{4}\left\{\epsilon\left(x^{-}-y^{-}\right)-\frac{x^{-}-y^{-}}{L}\right\} \delta_{i j} \delta^{(2)}\left(x^{\perp}-y^{\perp}\right),
$$

where each index $(i)$ stands for $\pi$ or $\sigma$. By making use of this commutation relation, it is shown that the creation and annihilation operators are simply constructed from the Fourier coefficients of $\varphi_{i}$ with respect to $x^{-}$:

$$
\varphi_{i}(x)=\sum_{n>0} \frac{1}{\sqrt{4 n \pi}}\left[a_{n}^{(i)}\left(x^{+}, x^{\perp}\right) e^{-i \frac{\pi n}{L} x^{-}}+a_{n}^{(i) \dagger}\left(x^{+}, x^{\perp}\right) e^{i \frac{n \pi}{L} x^{-}}\right],
$$

where the coefficients satisfy

$$
\left[a_{n}^{(i)}\left(x^{+}, x^{\perp}\right), a_{m}^{\dagger(j)}\left(x^{+}, y^{\perp}\right)\right]=\delta_{n, m} \delta_{i, j} \delta^{(2)}\left(x^{\perp}-y^{\perp}\right)
$$

in spite of the presence of interaction. The trivial Fock vacuum is defined as $a_{n}^{(i)}|0\rangle=0$ for any $n$. 
Instead of one zero-mode constraint (2.26) here we have two coupled zero-mode constraints

$$
\begin{aligned}
& \chi_{\pi} \equiv \frac{1}{2 L} \int_{-L}^{L} d x^{-}\left[\left(\mu^{2}-\partial_{\perp}^{2}\right) \pi+\lambda \pi\left(\pi^{2}+\sigma^{2}\right)\right]=0, \\
& \chi_{\sigma} \equiv \frac{1}{2 L} \int_{-L}^{L} d x^{-}\left[\left(\mu^{2}-\partial_{\perp}^{2}\right) \sigma+\lambda \sigma\left(\pi^{2}+\sigma^{2}\right)-c\right]=0
\end{aligned}
$$

which are also represented by

$$
\begin{aligned}
& \chi_{\pi}=-\frac{1}{2 L} \int_{-L}^{L} d x^{-} 2 \partial_{+} \partial_{-} \pi=0 \\
& \chi_{\sigma}=-\frac{1}{2 L} \int_{-L}^{L} d x^{-} 2 \partial_{+} \partial_{-} \sigma=0
\end{aligned}
$$

through the equation of motion (see (2.27)).

Our next task is to solve these constraints within some approximation. As explained in the beginning of this section, it is sufficient to obtain a solution corresponding to the perturbation theory around the classical (tree level) SSB vacuum in equal-time quantization. For this purpose it is convenient to further divide the zero modes as

$$
\begin{aligned}
& \pi_{0}=v_{\pi}+\omega_{\pi}, \\
& \sigma_{0}=v_{\sigma}+\omega_{\sigma},
\end{aligned}
$$

where $v_{\pi}$ and $v_{\sigma}$ are the classical constant pieces and $\omega_{\pi}$ and $\omega_{\sigma}$ are their operator parts. Then the zero-mode constraints are split into the classical and the operator parts. The classical parts of the zero-mode constraints are given by

$$
\begin{aligned}
& \mu^{2} v_{\pi}+\lambda v_{\pi}^{3}+\lambda v_{\pi} v_{\sigma}^{2}=0 \\
& \mu^{2} v_{\sigma}+\lambda v_{\sigma}^{3}+\lambda v_{\sigma} v_{\pi}^{2}=c,
\end{aligned}
$$

which are nothing but the condition determining the minima of the classical potential. The conventional choice of the solution is $v_{\pi}=0$ and $v_{\sigma}=v$, where $\mu^{2} v+\lambda v^{3}=c$. 
The operator zero modes are solved by substituting the perturbative expansion

$$
\omega_{i}=\sum_{k=1} \lambda^{k} \omega_{i}^{(k)}
$$

into the operator constraints. The nontrivial problem which we encounter in solving them is the choice of operator ordering, especially the ordering between the zero modes and the nonzero modes. As mentioned in the Sec 2, we assume the Weyl ordering. In the present context this ordering has an advantage that it gives a correct description of the symmetric phase $\left[\mu^{2}>0, c \rightarrow 0\right]$ from the view point of equal-time quantization. This is closely examined in Appendix C.

The operator part of the zero-mode constraints are explicitly written down under the Weyl ordering as follows:

$$
\begin{aligned}
\left(-m_{\pi}^{2}+\partial_{\perp}^{2}\right) \omega_{\pi} & =\frac{\lambda}{2 L} \int_{-L}^{L} d x^{-}\left(\varphi_{\pi}^{3}+\varphi_{\pi} \varphi_{\sigma}^{2}+2 v \varphi_{\pi} \varphi_{\sigma}\right) \\
& +\frac{\lambda}{2 L} \int_{-L}^{L} d x^{-}\left\{\left(\omega_{\pi} \varphi_{\pi}^{2}+\varphi_{\pi}^{2} \omega_{\pi}+\varphi_{\pi} \omega_{\pi} \varphi_{\pi}\right)+\frac{1}{2}\left(\omega_{\pi} \varphi_{\sigma}^{2}+\varphi_{\sigma}^{2} \omega_{\pi}\right)\right. \\
& \left.+\frac{1}{2}\left(\omega_{\sigma} \varphi_{\sigma} \varphi_{\pi}+\varphi_{\pi} \omega_{\sigma} \varphi_{\sigma}+\varphi_{\sigma} \omega_{\sigma} \varphi_{\pi}+\varphi_{\sigma} \varphi_{\pi} \omega_{\sigma}\right)\right\} \\
& +\lambda\left(\omega_{\pi}^{3}+\frac{1}{2} \omega_{\pi} \omega_{\sigma}^{2}+\frac{1}{2} \omega_{\sigma}^{2} \omega_{\pi}+v \omega_{\pi} \omega_{\sigma}+v \omega_{\sigma} \omega_{\pi}\right) \\
\left(-m_{\sigma}^{2}+\partial_{\perp}^{2}\right) \omega_{\sigma} & =\frac{\lambda}{2 L} \int_{-L}^{L} d x^{-}\left(\varphi_{\sigma}^{3}+\varphi_{\sigma} \varphi_{\pi}^{2}+v \varphi_{\pi}^{2}+3 v \varphi_{\sigma}^{2}\right) \\
& +\frac{\lambda}{2 L} \int_{-L}^{L} d x^{-}\left\{\left(\omega_{\sigma} \varphi_{\sigma}^{2}+\varphi_{\sigma}^{2} \omega_{\sigma}+\varphi_{\sigma} \omega_{\sigma} \varphi_{\sigma}\right)+\frac{1}{2}\left(\omega_{\sigma} \varphi_{\pi}^{2}+\varphi_{\pi}^{2} \omega_{\sigma}\right)\right. \\
& \left.+\frac{1}{2}\left(\omega_{\pi} \varphi_{\sigma} \varphi_{\pi}+\varphi_{\pi} \omega_{\pi} \varphi_{\sigma}+\varphi_{\sigma} \omega_{\pi} \varphi_{\pi}+\varphi_{\sigma} \varphi_{\pi} \omega_{\pi}\right)\right\} \\
& +\lambda\left(\omega_{\sigma}^{3}+\frac{1}{2} \omega_{\sigma} \omega_{\pi}^{2}+\frac{1}{2} \omega_{\pi}^{2} \omega_{\sigma}+v \omega_{\pi}^{2}+3 v \omega_{\sigma}^{2}\right)
\end{aligned}
$$

where each mass term is defined as $m_{\sigma}^{2}=\mu^{2}+3 \lambda v^{2}$ and $m_{\pi}^{2}=\mu^{2}+\lambda v^{2}$, respectively. Here it is worth referring to the scaling property of the zero mode with respect to $x^{-}$direction. The explicit form of the constraints shows that the zero modes are implicitly composed of 
the complicated combination of the following type of integrals

$$
\frac{1}{2 L} \int_{-L}^{L} d x^{-} \varphi_{i}^{n}(x, L) \varphi_{j}^{m}(x, L)
$$

where the explicit $L$ dependence is labeled and $n, m$ are some non-negative integers. The important feature of (4.17) is its invariance under the scale transformation $L \rightarrow s L$,

$$
\frac{1}{2 s L} \int_{-s L}^{s L} d x^{-} \varphi_{i}^{n}(x, s L) \varphi_{j}^{m}(x, s L)=\frac{1}{2 L} \int_{-L}^{L} d x^{-} \varphi_{i}^{n}(x, L) \varphi_{j}^{m}(x, L)
$$

which is on account of the relation

$$
\varphi(s x, s L)=\varphi(x, L)
$$

derived from (4.3) or (4.4). Thus the zero modes have no explicit dependence on the box size $L$ and the naive continuum limit $L \rightarrow \infty$ may be safely taken at least for the zero-mode sector.

Using the explicit form of the zero-mode constraints, the lowest order solution of the perturbative zero modes for $\omega_{\pi}$ and $\omega_{\sigma}$ is now easily obtained [15]:

$$
\begin{aligned}
\left(-m_{\pi}^{2}+\partial_{\perp}^{2}\right) \omega_{\pi}^{(1)} & =\frac{\lambda}{2 L} \int_{-L}^{L} d x^{-}\left(\varphi_{\pi}^{3}+\varphi_{\pi} \varphi_{\sigma}^{2}+2 v \varphi_{\pi} \varphi_{\sigma}\right) \\
\left(-m_{\sigma}^{2}+\partial_{\perp}^{2}\right) \omega_{\sigma}^{(1)} & =\frac{\lambda}{2 L} \int_{-L}^{L} d x^{-}\left(\varphi_{\sigma}^{3}+\varphi_{\sigma} \varphi_{\pi}^{2}+v \varphi_{\pi}^{2}+3 v \varphi_{\sigma}^{2}\right)
\end{aligned}
$$

where there is no operator-ordering ambiguity between the zero modes and the non-zero modes. Let us briefly see the feature of these explicit solutions. One can find that there is no divergence in $\omega_{\pi}^{(1)}$ due to the positivity of longitudinal momentum. Moreover this feature is valid beyond the leading approximation, that is, $\omega_{\pi}^{(i)}$ has no divergence for any $i \in \mathbf{N}$, and leads to

$$
\langle\pi\rangle=\left\langle\sum_{i=1}^{\infty} \omega_{\pi}^{(i)}\right\rangle+\left\langle\varphi_{\pi}\right\rangle=0
$$

which is expected from the equal-time perturbation theory. We will see that this well-defined zero mode solution is used in the actual calculation. On the other hand, $\omega_{\sigma}^{(1)}$ contains the 
divergence which is similar to the tadpole divergence in the equal-time perturbation theory. This divergence is essentially the same as the one discussed by Robertson [13] in the discrete symmetry breaking of $\phi_{2}^{4}$ model and can be formally renormalized into $v$ through the mass renormalization.

We are now in a position to examine the consequence of the explicit solutions (4.20) and (4.21). In order to emphasize the importance of $c$, we first examine the case $c \equiv 0$ (or $\left.m_{\pi} \equiv 0\right)$ again which turns out to have internal inconsistency. We will then study the case $c \neq 0$ (or $m_{\pi} \neq 0$ ), $c \rightarrow 0$ and show that the singular behavior of the zero mode (3.7) recovers the correct $\sigma \pi \pi$ vertex [15].

(I) $c \equiv 0$

The equation of motion for $\pi$ is given by

$$
\begin{aligned}
\square \pi(x) & =j_{\pi}(x) \\
& \equiv-\lambda\left(\pi^{3}+\pi \sigma^{2}+2 v \pi \sigma^{\prime}\right),
\end{aligned}
$$

where $\sigma^{\prime}=\sigma-v$ and $v=\sqrt{-\mu^{2} / \lambda}$. Rewriting (‥2) in the present context leads to

$$
\begin{aligned}
(2 \pi)^{3} \delta^{(3)}(\vec{q})\left\langle\pi\left|j_{\pi}(0)\right| \sigma\right\rangle & =\int d^{3} \vec{x}\left\langle\pi\left|\left(2 \partial_{+} \partial_{-}-\partial_{\perp}^{2}\right) \pi\right| \sigma\right\rangle \\
& =-\int d^{3} \vec{x}\left\langle\pi\left|\chi_{\pi}(x)\right| \sigma\right\rangle=0
\end{aligned}
$$

where $\vec{q}=\vec{q}_{\sigma}-\vec{p}_{\pi}$ is the momentum of the NG boson. This relation leads to an internal inconsistency: the NG-boson emission vertex $\sigma \rightarrow \pi \pi$ at $q^{2}=0$ is vanishing due to the r.h.s. of (4.24) or equivalently the zero-mode constraint $\chi_{\pi}=0$, while the l.h.s. of (4.24) gives the non-vanishing result as will be shown later.

Furthermore this inconsistency is connected with the current conservation which also means the charge conservation:

$$
\begin{aligned}
\dot{Q} & =\frac{d}{d x^{+}} \int d^{3} \vec{y} J^{+} \\
& =-\int d^{3} \vec{y}\left(\partial_{-} J^{-}+\partial_{\perp} J^{\perp}\right)
\end{aligned}
$$




$$
=-\int d^{2} y^{\perp}\left\{J^{-}\left(x^{-}=+L\right)-J^{-}\left(x^{-}=-L\right)\right\}=0 \quad,
$$

where the periodic boundary condition was used. In our model we have an explicit form of the $\mathrm{O}(2)$ current

$$
J^{\mu}=\partial_{\mu} \sigma \pi-\partial_{\mu} \pi \sigma
$$

The LF charge defined by $Q=\int d^{3} \vec{x} J^{+}$reduces to

$$
Q=\int d^{3} \vec{x}\left(\partial_{-} \varphi_{\sigma} \varphi_{\pi}-\partial_{-} \varphi_{\pi} \varphi_{\sigma}\right)
$$

which contains only the oscillating modes, because the operator part of the zero mode in addition to the pole term is dropped by the integration over $x^{-}$[5]. Thus the LF charge is well-defined even in the NG phase and always annihilates the vacuum simply by the $P^{+}$ conservation:

$$
Q|0\rangle=0
$$

which supports the trivial property of the LF vacuum. This will also be checked in later discussions, see (4.41) and (4.43).

The charge conservation can also be checked through the explicit calculation including the zero modes. In fact the straightforward but tedious calculation using the perturbative solution of the zero modes leads to

$$
\left[Q, P^{-}\right]=i \int d^{3} \vec{x}\left(v \chi_{\pi}+\omega_{\sigma} \chi_{\pi}-\omega_{\pi} \chi_{\sigma}\right)+(\text { divergence })
$$

where the divergence arises from the operator ordering and should be renormalized in an appropriate way. If we simply neglect this divergence, or the commutator is understood as Dirac bracket, the r.h.s. of (4.29) becomes zero owing to the zero-mode constraints.

By substituting $\sigma=\sigma^{\prime}+v$ into (4.26), we have

$$
J^{\mu}=\widehat{J}^{\mu}-v \partial_{\mu} \pi
$$


where $\widehat{J}^{\mu}=\partial_{\mu} \sigma^{\prime} \pi-\partial_{\mu} \pi \sigma^{\prime}$. From the current conservation, we obtain

$$
\begin{aligned}
\left\langle\pi\left|\int d^{3} \vec{x} \partial_{\mu} \widehat{J}^{\mu}(x)\right| \sigma\right\rangle_{x^{+}=0} & =-v\left\langle\pi\left|\int d^{3} \vec{x} \chi_{\pi}\right| \sigma\right\rangle_{x^{+}=0} \\
& =-i(2 \pi)^{3} \delta^{(3)}(\vec{q}) \frac{m_{\sigma}^{2}-m_{\pi}^{2}}{2 p_{\sigma}^{+}}\left\langle\pi\left|\widehat{J}^{+}(0)\right| \sigma\right\rangle,
\end{aligned}
$$

which means $\left\langle\pi\left|\widehat{J}^{+}(0)\right| \sigma\right\rangle=0\left(m_{\sigma}^{2} \neq m_{\pi}^{2}\right)$ due to the charge conservation or the zero-mode constraint. The charge conservation does not lead to the NG phase at all.

This pathology suggests that the zero-mode constraint without mass term is ill-defined. Up to the operator ordering, the zero-mode constraint for $\omega_{\pi}$ with $m_{\pi} \equiv 0$ is given by

$$
\begin{aligned}
\partial_{\perp}^{2} \omega_{\pi} & =\frac{\lambda}{2 L} \int_{-L}^{L} d x^{-}\left(\varphi_{\pi}^{3}+\varphi_{\pi} \varphi_{\sigma}^{2}+2 v \varphi_{\pi} \varphi_{\sigma}\right) \\
& +\frac{\lambda}{2 L} \int_{-L}^{L} d x^{-}\left(\omega_{\pi} \varphi_{\sigma}^{2}+2 \omega_{\sigma} \varphi_{\sigma} \varphi_{\pi}+3 \omega_{\pi} \varphi_{\pi}^{2}\right) \\
& +\lambda\left(\omega_{\pi}^{3}+\omega_{\pi} \omega_{\sigma}^{2}+2 v \omega_{\pi} \omega_{\sigma}\right)
\end{aligned}
$$

Note that it is not $\omega_{\pi}$ but $\int d^{3} \vec{x} \omega_{\pi}$ which is used to calculate the $\sigma \pi \pi$ vertex. Thus the real quantity to be considered is the integration of (4.32) over the LF which has the similar structure as that in two dimensions, because the l.h.s. of (4.32) vanishes by the transverse integration. However, in two dimensions, the zero-mode constraint without mass term is ill-defined in the interacting theory. The reason can be easily understood as follows. The overall factor $\lambda$ appearing on the r.h.s. of (4.32) is canceled and dropped out. The solution $\omega_{\pi}$ is then independent of $\lambda$. The Eq. (4.32) (before the transverse integration) dictates that $\omega_{\pi}$ does depend on $\lambda$. Therefore it is necessary to introduce the NG-boson mass to make the theory well-defined.

(II) $c \neq 0$ and $c \rightarrow 0$

Having the explicit breaking NG boson mass, we now derive the tree-level matrix element for the $\sigma \pi \pi$ scattering [15]. The singular behavior of the NG-boson zero mode proposed in the previous section is in fact derived from $(4.20)$ :

$$
\lim _{m_{\pi}^{2} \rightarrow 0} m_{\pi}^{2} \int d^{3} \vec{x} \omega_{\pi}^{(1)}=-\lambda \int d^{3} \vec{x}\left(\varphi_{\pi}^{3}+\varphi_{\pi} \varphi_{\sigma}^{2}+2 v \varphi_{\pi} \varphi_{\sigma}\right) \neq 0
$$


which is not restricted to the lowest order but is valid for higher order solutions:

$$
\int d^{3} \vec{x} \omega_{\pi}^{(n)} \sim \frac{1}{m_{\pi}^{2}} .
$$

See Appendix D for more details.

This actually ensures the correct $\sigma \rightarrow \pi \pi$ vertex as well as the non-vanishing current vertex. Indeed the $\sigma \rightarrow \pi \pi$ vertex at $q^{2}=0$ is evaluated as follows:

$$
\begin{aligned}
\left\langle\pi, \pi\left(q^{2}=0\right) \mid \sigma\right\rangle & =\lim _{m_{\pi}^{2} \rightarrow 0} i \int d^{4} x e^{i q x}\left\langle\pi\left|\left(\square+m_{\pi}^{2}\right) \pi(x)\right| \sigma\right\rangle \\
& =\lim _{m_{\pi}^{2} \rightarrow 0} i \int d x^{+} e^{i q^{-} x^{+}}\left\langle\pi\left|m_{\pi}^{2} \int_{-\infty}^{+\infty} d^{3} \vec{x} \omega_{\pi}^{(1)}\right| \sigma\right\rangle \\
& =i(2 \pi)^{4} \delta\left(q^{-}+p_{\pi}^{-}-p_{\sigma}^{-}\right) \delta^{(3)}\left(\vec{p}_{\sigma}-\vec{p}_{\pi}\right)(-2 \lambda v)
\end{aligned}
$$

where $q^{\mu}=p_{\sigma}^{\mu}-p_{\pi}^{\mu}$ is the four momentum of the NG boson. The current vertex is also obtained by using the operator relation

$$
\partial_{\mu} J^{\mu}(x)=v m_{\pi}^{2} \pi(x)
$$

which leads to

$$
\partial_{\mu} \widehat{J}^{\mu}(x)=v\left(\square+m_{\pi}^{2}\right) \pi(x)=v j_{\pi}(x)
$$

where $j_{\pi}(x)=-\lambda\left(\pi^{3}+\pi \sigma^{2}+2 v \pi \sigma^{\prime}\right)$ with $\sigma^{\prime}=\sigma-v$. Then we can confirm the following relations:

$$
\begin{aligned}
\left\langle\pi\left|\int d^{3} \vec{x} \partial_{\mu} \widehat{J}^{\mu}(x)\right| \sigma\right\rangle & =\int d^{3} \vec{x}\left\langle\pi\left|v j_{\pi}(x)\right| \sigma\right\rangle \\
& =v m_{\pi}^{2}\left\langle\pi\left|\int d^{3} \vec{x} \omega_{\pi}^{(1)}(x)\right| \sigma\right\rangle \\
& =-2 \lambda v^{2}(2 \pi)^{3} \delta\left(q^{+}\right) \delta^{(2)}\left(q^{\perp}\right)
\end{aligned}
$$

in the limit of $m_{\pi} \rightarrow 0$ or $v \rightarrow \sqrt{\frac{-\mu^{2}}{\lambda}}$. Throughout the calculations we have used the covariant normalization of states $\left\langle p_{\beta} \mid p_{\alpha}\right\rangle=(2 \pi)^{3} 2 p_{\alpha}^{+} \delta^{(3)}\left(\vec{p}_{\alpha}-\vec{p}_{\beta}\right)$ and the on-shell mode expansion for $\varphi$ in the continuum limit. 
Let us see the property of the LF charge in this case $(c \neq 0)$. As already mentioned, the LF charge is well-defined even in the NG phase and always annihilates the vacuum simply by the $P^{+}$conservation:

$$
Q|0\rangle=0 \quad,
$$

which supports the trivial property of the LF vacuum. The trivial LF vacuum is also consistent with the explicit computation of the commutators. We can show that the oscillating modes are transformed under the action of $Q$ as

$$
\left[Q, \varphi_{\sigma}\right]=-i \varphi_{\pi} \quad \text { and } \quad\left[Q, \varphi_{\pi}\right]=i \varphi_{\sigma}
$$

where use has been made of (4.3) and (4.27). Then we have

$$
\left\langle\left[Q, \varphi_{\sigma}\right]\right\rangle=\left\langle\left[Q, \varphi_{\pi}\right]\right\rangle=0
$$

which is consistent with the trivial vacuum: $Q|0\rangle=0$. On the other hand, the lowest order solution of the zero modes yields rather complicated transformation property of the zero modes:

$$
\begin{aligned}
& {\left[Q, \omega_{\sigma}\right]=\left(-m_{\sigma}^{2}+\partial_{\perp}^{2}\right)^{-1} \frac{i \lambda}{2 L} \int_{-L}^{L} d x^{-}\left(\varphi_{\pi}^{3}+\varphi_{\pi} \varphi_{\sigma}^{2}+4 v \varphi_{\pi} \varphi_{\sigma}\right) \neq-i \omega_{\pi}} \\
& {\left[Q, \omega_{\pi}\right]=\left(-m_{\pi}^{2}+\partial_{\perp}^{2}\right)^{-1} \frac{i \lambda}{2 L} \int_{-L}^{L} d x^{-}\left(\varphi_{\sigma}^{3}+\varphi_{\sigma} \varphi_{\pi}^{2}-2 v \varphi_{\pi}^{2}+2 v \varphi_{\sigma}^{2}\right) \neq i \omega_{\sigma}}
\end{aligned}
$$

Nevertheless, it is straightforward to confirm that

$$
\langle[Q, \pi]\rangle=\langle[Q, \sigma]\rangle=0
$$

by putting together (4.40) and (4.42). Eq. (4.43) can also be checked by use of the commutator for the full fields (2.29) or (2.31) up to operator ordering: Would-be non-vanishing term arising from the sign function is precisely cancelled by that from the extra term in the commutator. Then we conclude that our LF charge does annihilate the vacuum in accord with the general argument [5] for the trivial vacuum based on the zero-mode constraint 
through which the zero mode can be solved away out of the physical Fock space. We should remark that $[Q, \pi] \neq i \sigma$ and $[Q, \sigma] \neq-i \pi$ even in the $m_{\pi} \rightarrow 0$ limit which are on account of the effect of zero modes. They are contrasted to those in the usual equal-time case: $\left[Q^{\mathrm{et}}, \sigma\right]=-i \pi$, and $\left[Q^{\mathrm{et}}, \pi\right]=i \sigma$. Since the information of the equal-time vacuum symbolically denoted by " $Q$ et $|0\rangle_{\text {et }} \neq 0$ " is expected to be carried into the zero mode in DLCQ, the unusual transformation property of the zero modes in the NG phase seems to be natural.

Finally, we can show from (4.38) that the regularized zero mode leads to non-conservation of the LF charge in the symmetric limit of $m_{\pi}^{2} \rightarrow 0$ [15]:

$$
\dot{Q}=\frac{1}{i}\left[Q, P^{-}\right]=v \lim _{m_{\pi}^{2} \rightarrow 0} m_{\pi}^{2} \int d^{3} \vec{x} \omega_{\pi} \neq 0
$$

Therefore the SSB in DLCQ is realized as if it were an explicit symmetry breaking. Actually, there exists no NG theorem on the LF. Instead, the singular behavior (4.34) establishes existence of the massless NG boson coupled to the current whose charge satisfies $Q|0\rangle=0$ and $\dot{Q} \neq 0$, in much the same as the NG theorem in the equal-time quantization which ensures existence of the massless NG boson coupled to the current whose charge satisfies $Q^{\mathrm{et}}|0\rangle_{\mathrm{et}} \neq 0$ and $\dot{Q^{\mathrm{et}}}=0$.

\section{2 $N N \pi$ vertex}

As was shown in the previous subsection, the classical part of the zero mode for $\sigma$ is given by $v$ which contributes to the vacuum expectation value $\langle\sigma\rangle=v$ in the trivial LF vacuum. Rewriting the Lagrangian (4.1) by the shifted field $\sigma^{\prime}=\sigma-v$, we find the standard Lagrangian in the broken phase

$$
\begin{aligned}
\mathcal{L} & =\bar{\psi}\left\{i \gamma^{\mu} \partial_{\mu}-M(x)\right\} \psi+\frac{1}{2}\left(\partial_{\mu} \sigma^{\prime}\right)^{2}+\frac{1}{2}\left(\partial_{\mu} \pi\right)^{2} \\
& -\frac{1}{2} m_{\sigma}^{2} \sigma^{2}-\frac{1}{2} m_{\pi}^{2} \pi^{2}-\frac{\lambda}{4}\left(\sigma^{\prime}\right)^{4}-\frac{\lambda}{4}(\pi)^{4}-\lambda v\left(\sigma^{\prime}\right)^{3}-\lambda v \sigma^{\prime} \pi^{2}-\frac{\lambda}{2}\left(\sigma^{\prime}\right)^{2} \pi^{2}
\end{aligned}
$$


where $M(x)=m_{\psi}+g\left(\sigma^{\prime}(x)+i \gamma_{5} \pi(x)\right)$ and the "nucleon" field $\psi$ acquired the "degenerate" mass $m_{\psi}=g v$.

Let us first clarify the fermion contribution to the NG-boson zero mode. By integrating the equation of motion over $x^{-}$,

$$
\left(-2 \partial_{+} \partial_{-}+\partial_{\perp}^{2}-m_{\pi}^{2}\right) \pi-\lambda\left\{\pi^{3}+\pi\left(\sigma^{\prime}\right)^{2}+2 v \sigma^{\prime} \pi\right\}-g \bar{\psi} i \gamma_{5} \psi=0
$$

we can easily derive the zero-mode constraint for the NG boson:

$$
\left(-m_{\pi}^{2}+\partial_{\perp}^{2}\right) \omega_{\pi}=\frac{g}{2 L} \int_{-L}^{L} d x^{-} \bar{\psi} i \gamma_{5} \psi+(\text { scalar and pseudo-scalar parts })
$$

Henceforth we shall omit both scalar and pseudo-scalar parts for simplicity.

As we have seen in the $\sigma \pi \pi$ vertex, the $N N \pi$ vertex at $q^{2}=0$ is essentially given by

$$
-m_{\pi}^{2} \int d^{3} \vec{x} \omega_{\pi}=g \int d^{3} \vec{x} \bar{\psi} i \gamma_{5} \psi
$$

which is consistent to our proposal (3.7) as long as the r.h.s. is non-vanishing. Let us estimate the r.h.s. of (4.48) in detail. We assume the anti-periodic boundary condition for the fermion field and neglect the fermion zero mode. Introducing the projection operator $\Lambda_{( \pm)}=\frac{1}{\sqrt{2}} \gamma^{0} \gamma^{ \pm}$ where $\gamma^{ \pm}=\frac{1}{\sqrt{2}}\left(\gamma^{0} \pm \gamma^{3}\right)$, the fermion field can be decomposed into the dynamical plus the nondynamical components $\psi=\psi_{(+)}+\psi_{(-)}$where $\psi_{( \pm)}=\Lambda_{( \pm)} \psi$. Note that $\left(\gamma^{+}\right)^{2}=\left(\gamma^{-}\right)^{2}=0$ implies $\Lambda_{(+)} \Lambda_{(-)}=\Lambda_{(-)} \Lambda_{(+)}=0$. The relevant part of the Lagrangian can be written in terms of the two kinds of fermion projections:

$$
\begin{aligned}
\mathcal{L} & =\bar{\psi}(x)\left\{i \gamma^{\mu} \partial_{\mu}-M(x)\right\} \psi(x) \\
& =i \sqrt{2} \psi_{(+)}^{\dagger} \partial_{+} \psi_{(+)}+i \sqrt{2} \psi_{(-)}^{\dagger} \partial_{-} \psi_{(-)} \\
& +\psi_{(+)}^{\dagger}\left(i \gamma^{0} \gamma^{k} \partial_{k}-\gamma^{0} M\right) \psi_{(-)}+\psi_{(-)}^{\dagger}\left(i \gamma^{0} \gamma^{k} \partial_{k}-\gamma^{0} M\right) \psi_{(+)}
\end{aligned}
$$

The equation of motion for $\psi_{(-)}$leads to the constraint equation

$$
i \sqrt{2} \partial_{-} \psi_{(-)}+\left(i \gamma^{0} \gamma^{k} \partial_{k}-\gamma^{0} M\right) \psi_{(+)}=0
$$


whose solution is readily obtained as

$$
\psi_{(-)}(x)=\frac{i}{2 \sqrt{2}} \int_{-L}^{L} d y^{-} \epsilon\left(x^{-}-y^{-}\right)\left\{i \gamma^{0} \gamma^{k} \partial_{k}-\gamma^{0} M(y)\right\} \psi_{(+)}(y)
$$

By substituting the solution of $\psi_{(-)}$into the r.h.s. of (4.48), one obtains

$$
\int d^{3} \vec{x} \bar{\psi} i \gamma_{5} \psi=\frac{1}{2 \sqrt{2}} \int d^{3} \vec{x} d y^{-} \epsilon\left(x^{-}-y^{-}\right) \psi_{(+)}^{\dagger}(x)\left\{M(x) \gamma_{5}-\gamma_{5} M(y)\right\} \psi_{(+)}(y)
$$

where the r.h.s. is non-vanishing as is seen from the explicit form of $M(x)=m_{\psi}+g \sigma^{\prime}(x)+$ $i g \gamma_{5} \pi(x)$. Thus our proposal for the singular behavior of the NG-boson zero mode is confirmed.

It is worth mentioning that exceptional situation happens for the tree-level $N N \pi$ coupling, in which case we should keep only the $x$-independent part of $M(x)$, i.e., $m_{\psi}$ in (4.52), because the rest gives higher order contributions. Keeping only $m_{\psi}$ is equivalent to applying the free equation of motion to the r.h.s. of (4.48). It is a well-known peculiarity on the LF that the pseudo-scalar density for free massive fermion, when integrated over the LF, vanishes. This is what the r.h.s. of (4.52) implies for $g=0$ and $M=m_{\psi}$. Owing to this specific property on the LF, the physical amplitude for the tree-level $N N \pi$ scattering at $q^{2}=0$ becomes zero, which is of course consistent with that in the equal-time quantization. Note that $q^{2}=0$ is nothing but the soft momentum limit $q^{\mu}=0$ for the "degenerate nucleon mass" and the physical amplitude of the NG-boson emission vertex as well as the associated current vertex is known to be zero in such a limit for kinematical reason from the low energy theorem, even when $N N \pi$ coupling constant and $g_{A}$ are non-zero [23].

\section{Zero-Mode Problem in the Continuum LF Quanti- zation}

The issue of symmetry breakings is important not only in the DLCQ but also in the continuum LF framework such as the renormalization-group approach. Wilson et al. [3] studied the sigma model "without zero mode" in the continuum framework and described the broken 
phase at the tree level by constructing the corresponding "effective Hamiltonian" without zero mode and with the "unusual counter terms" which compensate the "removal of the zero mode". Instead of comparing our result with theirs in a direct manner, we here examine the same sigma model in the broken phase in the general continuum framework, paying special attention to the boundary condition. As we emphasized in Section 2 (also in Appendix B), the boundary condition in the LF quantization contains dynamical information and is crucial to define the theory. Then we shall demonstrate that it is actually impossible to remove the zero mode in the continuum theory in a manner consistent with the trivial vacuum. The point is that the real problem with the zero mode in the continuum theory is not a single mode with $p^{+} \equiv 0$, which is just measure zero, but the accumulating point $p^{+} \rightarrow 0$ [17. This is in sharp contrast to our result in DLCQ where the trivial vacuum is always guaranteed thanks to the clean separation and explicit removal of the zero mode through the zero-mode constraint.

Let us illustrate this by starting with the canonical commutator for the fields $\sigma, \pi$ in the bosonic part of the $O(2)$ sigma model (4.2) (without explicit symmetry breaking term, $c \equiv 0)$ in the continum theory:

$$
\begin{aligned}
& {[\sigma(x), \sigma(y)]_{x^{+}=y^{+}}=-\frac{i}{4} \epsilon\left(x^{-}-y^{-}\right) \delta^{(2)}\left(x^{\perp}-y^{\perp}\right)} \\
& {[\pi(x), \pi(y)]_{x^{+}=y^{+}}=-\frac{i}{4} \epsilon\left(x^{-}-y^{-}\right) \delta^{(2)}\left(x^{\perp}-y^{\perp}\right)}
\end{aligned}
$$

where the sign function

$$
\epsilon\left(x^{-}\right)=\frac{i}{\pi} \mathcal{P} \int_{-\infty}^{+\infty} \frac{d p^{+}}{p^{+}} e^{-i p^{+} x^{-}}
$$

is defined by the principal value prescription and hence has no $p^{+} \equiv 0$ mode but does have an accumulating point $p^{+} \rightarrow 0$. This accumulating point is really a trouble as we will see in the followings. Then, as far as we use this sign function for the commutator, we cannot really remove the zero mode in this sense.

We first look at the transformation property of the fields $\sigma, \pi$. The conserved current 
associated with the symmetry of the Lagrangian is given by

$$
J_{\mu}=\pi \partial_{\mu} \sigma-\sigma \partial_{\mu} \pi
$$

and the LF charge is defined by

$$
Q=\int d^{3} \vec{x}\left(\pi \partial_{-} \sigma-\sigma \partial_{-} \pi\right)
$$

From the canonical commutation relations (5.1) and (5.2) we can easily find

$$
\begin{aligned}
& {[Q, \sigma(x)]=-i \pi(x)+\frac{i}{4}\left[\pi\left(x^{-}=\infty\right)+\pi\left(x^{-}=-\infty\right)\right],} \\
& {[Q, \pi(x)]=i \sigma(x)-\frac{i}{4}\left[\sigma\left(x^{-}=\infty\right)+\sigma\left(x^{-}=-\infty\right)\right] .}
\end{aligned}
$$

To obtain a sensible transformation property of the fundamental fields, the surface terms must vanish as operators:

$$
\pi\left(x^{-}=\infty\right)+\pi\left(x^{-}=-\infty\right)=\sigma\left(x^{-}=\infty\right)+\sigma\left(x^{-}=-\infty\right)=0 .
$$

However, this condition, anti-periodic boundary condition, means that the zero mode is not allowed to exist and hence its classical part, condensate $\langle\sigma\rangle$, does not exist at all. Thus we have no spontaneous symmetry breaking contrary to the initial assumption.

We then seek for a modification of the boundary condition to save the condensate and vanishing surface term simultaneously. The lesson from the above argument is that we cannot impose the canonical commutation relation for the full fields, because then not only the surface term but also the zero mode (and hence condensate) are required to vanish due to the relation (5.8). So, let us first separate the constant part or condensate (classical zero mode) $v$ from $\sigma$ and then impose the canonical commutation relations for the fields without zero modes, $\pi$ and the shifted field $\phi=\sigma-v=\sigma^{\prime}$ (here we use $\phi$ instead of $\sigma^{\prime}$ ), which are now consistent with the anti-periodic boundary condition and (5.8). This actually corresponds to the usual quantization around the classical SSB vacuum in the equal-time quantization. 
The constant part $v$ should be understood to be determined by the minimum of the classical potential

$$
\begin{aligned}
V & =\frac{1}{2} \mu^{2}\left(\sigma^{2}+\pi^{2}\right)+\frac{\lambda}{4}\left(\sigma^{2}+\pi^{2}\right)^{2} \\
& =\frac{1}{2} m_{\phi}^{2} \phi^{2}+\lambda v \phi\left(\phi^{2}+\pi^{2}\right)+\frac{\lambda}{4}\left(\phi^{2}+\pi^{2}\right)^{2}
\end{aligned}
$$

where $v=\sqrt{-\mu^{2} / \lambda}, \mu^{2}<0$ and $m_{\phi}^{2}=2 \lambda v^{2}$. In the renormalization group approach, the potential (5.9) appears as an "effective Hamiltonian" [3], while the same potential can be obtained simply through shifting $\sigma$ to $\phi=\sigma-v$. The canonical commutation relation for $\sigma$ is now replaced by

$$
[\phi(x), \phi(y)]_{x^{+}=y^{+}}=-\frac{i}{4} \epsilon\left(x^{-}-y^{-}\right) \delta^{(2)}\left(x^{\perp}-y^{\perp}\right)
$$

Now that the quantized fields have been arranged to obey the anti-periodic boundary condition, one might consider that we have removed the zero mode. It is not true, however, as far as we are using the commutator with the sign function, (5.10), in which the zero mode as an accumulating point is persistent to exist.

Let us look at the LF charge which is given by

$$
Q=\int d^{3} \vec{x}\left(\pi \partial_{-} \phi-\phi \partial_{-} \pi-v \partial_{-} \pi\right)
$$

The straightforward calculation leads to

$$
\begin{gathered}
{[Q, \phi(x)]=-i \pi(x)+\frac{i}{4}\left[\pi\left(x^{-}=\infty\right)+\pi\left(x^{-}=-\infty\right)\right],} \\
{[Q, \pi(x)]=i \phi(x)+\frac{i}{2} v-\frac{i}{4}\left[\phi\left(x^{-}=\infty\right)+\phi\left(x^{-}=-\infty\right)\right]}
\end{gathered}
$$

where the surface terms should vanish:

$$
\phi\left(x^{-}=\infty\right)+\phi\left(x^{-}=-\infty\right)=\pi\left(x^{-}=\infty\right)+\pi\left(x^{-}=-\infty\right)=0
$$

for the same reason as before. Thus we find

$$
[Q, \phi(x)]=-i \pi(x)
$$




$$
[Q, \pi(x)]=i \phi(x)+\frac{i}{2} v
$$

The constant term on the r.h.s. of (5.16) has its origin in the commutation relation (5.2), or equivalently,

$$
\left[\partial_{-} \pi(x), \pi(y)\right]=-\frac{i}{2} \delta^{(3)}(\vec{x}-\vec{y})
$$

Namely,

$$
\begin{aligned}
\langle 0|[Q, \pi(x)]| 0\rangle & =i\langle 0|\phi(x)| 0\rangle+\frac{i}{2} v, \\
& =\frac{i}{2} v \neq 0
\end{aligned}
$$

Then we find that the LF charge does not annihilate the vacuum $Q|0\rangle \neq 0$ and we have lost the trivial vacuum which is a vital feature of the LF quantization. There actually exist infinite number of zero-mode states $|\alpha\rangle \equiv e^{i \alpha Q}|0\rangle$ such that $P^{+}|\alpha\rangle=e^{i \alpha Q} P^{+}|0\rangle=0$, where we have used $\left[P^{+}, Q\right]=0$ and $\alpha$ is a real number: All these states satisfy the "Fock-vacuum condition" $a\left(p^{+}\right)|\alpha\rangle=0$ and hence the true unique vacuum cannot be specified by this condition in contrast to the usual expectation. This implies that the zero mode has not been removed, even though the Hamiltonian has been rearranged by shifting the field into the one without exact zero mode $p^{+} \equiv 0$. This is in sharp contrast to DLCQ in Sect.4 where the surface terms in (5.12) and (5.13) and the constant term in (5.16) do vanish altogether thanks to the additional term $-\left(x^{-}-y^{-}\right) / L$ ("subtraction of the zero mode") besides the sign function $\epsilon\left(x^{-}-y^{-}\right)$in the canonical commutator (4.3).

It should be noted that somewhat peculiar situation happens to the LF charge due to this boundary condition at the surface term: Although the local current is conserved, the LF charge is not. In fact, integrating the equation of the current conservation $\partial_{\mu} J^{\mu}=0$, we have

$$
\begin{aligned}
\frac{d Q}{d x^{+}} & =-\int d^{3} \vec{x}\left(\partial_{-} J^{-}+\partial_{\perp} J^{\perp}\right) \\
& =v \int d^{3} \vec{x} \partial_{+} \partial_{-} \pi
\end{aligned}
$$




$$
\begin{aligned}
& =v \int d^{2} x^{\perp}\left[\partial_{+} \pi\left(x^{-}=\infty\right)-\partial_{+} \pi\left(x^{-}=-\infty\right)\right] \\
& =2 v \int d^{2} x^{\perp} \partial_{+} \pi\left(x^{-}=\infty\right) \neq 0
\end{aligned}
$$

where the anti-periodic boundary condition (5.14) has been used. Thus in the continuum theory LF charge is not conserved in spite of the conservation of the local current. This charge non-conservation can also be checked by direct calculation:

$$
\begin{aligned}
i \frac{d Q}{d x^{+}} & =[Q, H] \\
& =-\frac{i}{2} \lambda v \int d^{3} \vec{x}\left[\pi\left(\phi^{2}+\pi^{2}\right)+2 v \phi \pi\right] \\
& =i v \int d^{2} x^{\perp}\left[\partial_{+} \pi\left(x^{-}=\infty\right)-\partial_{+} \pi\left(x^{-}=-\infty\right)\right]
\end{aligned}
$$

where use has been made of the equation of motion

$$
-2 \partial_{+} \partial_{-} \pi+\partial_{\perp}^{2} \pi=2 \lambda v \phi \pi+\lambda \pi\left(\phi^{2}+\pi^{2}\right)
$$

as well as the anti-periodic boundary condition (5.14).

The resulting Hamiltonian via the field shifting coincides with the "effective Hamiltonian" of Ref. [3] which was obtained by "removing the zero mode and adding unusual counter terms" for it. The above peculiarity of the LF charge, non-conservation of the LF charge and conservation of the local current, was also claimed in Ref.[3] for completely different reason than ours. They implicitly assumed vanishing surface terms altogether:

$$
\phi\left(x^{-}=\infty\right)=\phi\left(x^{-}=-\infty\right)=\pi\left(x^{-}=\infty\right)=\pi\left(x^{-}=-\infty\right)=0
$$

However, it is actually not allowed, because it contradicts the commutation relation (5.2) and (5.10). For instance, the commutation relation (5.17) yields

$$
\left[\int d^{3} \vec{x} \partial_{-} \pi(x), \pi(y)\right]_{x^{+}=y^{+}}=\int d^{3} \vec{x}\left[\partial_{-} \pi(x), \pi(y)\right]_{x^{+}=y^{+}}=-\frac{i}{2} \neq 0
$$

while (5.22) requires l.h.s. to vanish. If one illegitimately assumed (5.22) and neglected all surface terms, then the LF charge would have been conserved as is easily read off from (5.20), in contradiction to [3]. 
To summarize, in the general continuum LF quantization based on the canonical commutation relation with sign function, the LF charge does not annihilate the vacuum and is not conserved for the conserved local current. It corresponds to impossibility to remove the zero mode as an accumulating point in the continuum theory in a manner consistent with the trivial vacuum. Thus, in the continuum theory the greatest advantage of the LF quantization, the simplicity of the vacuum, is lost, although the NG-boson emission vertex can be nontrivial without such a manipulation as via the explicit NG boson mass in contrast to DLCQ.

Here it is worth suggesting that even in the continuum theory there exists a prescription, " $\nu$-theory" [17, which may give rise to the same result as that we obtained in DLCQ in this paper: the trivial vacuum $Q|0\rangle=0$, and the non-decoupling NG boson through the explicit breaking mass of the NG-boson or the singular behavior of the global zero mode of the NG boson. The $\nu$-theory modifies the sign function in the commutator into a certain function which vanishes at $x^{-}= \pm \infty$, by shaving the vicinity of the zero mode to tame the $1 / p^{+}$ singularity as $\left|p^{+}\right|^{\nu} / p^{+}(\nu>0)$. The limit of $\nu \rightarrow 0$ is taken only after whole calculation. Then there is no surface term nor constant term $\left(\frac{i}{2} v\right)$ in the commutator (5.12) and (5.13), and hence the transformation property of the fields and the trivial vacuum should be both realized. Also, the LF charge conservation is expected to follow unless we introduce the explicit symmetry breaking, which is in fact the same situation as in DLCQ. Thus, in order to realize the NG phase we could do the same game as DLCQ, namely, introduce the explicit breaking mass of the NG boson in such a way that the global zero mode of the NG boson behaves singular as $1 / m_{\pi}^{2}$ in the symmetric limit.

\section{Summary and Discussions}

We have studied how the continuous symmetry breaking in $(3+1)$-dimensions is described on the LF within the framework of DLCQ. We have shown that it is necessary to introduce 
an explicit symmetry-breaking mass of the NG boson $m_{\pi}$ in order to realize the NG phase in DLCQ. The NG phase is reproduced in the limit of $m_{\pi} \rightarrow 0$, where the peculiar behavior of the NG-boson zero mode is derived: The NG-boson zero mode, when integrated over the LF, must behave as $\sim 1 / m_{\pi}^{2}$. This ensures the non-vanishing matrix elements associated with the NG boson. However we encounter as an inevitable consequence that the LF charge is not conserved or even the current conservation breaks down even in the limit of $m_{\pi} \rightarrow 0$.

Here we emphasize that the $N G$ theorem does not exist on the $L F$. Instead we found the singular behavior (3.7) which in fact establishes existence of the massless NG boson coupled to the current such that $Q|0\rangle=0$ and $\dot{Q} \neq 0$, quite analogously to the NG theorem in the equal-time quantization which proves existence of the massless NG boson coupled to the current such that $Q^{\text {et }}|0\rangle_{\text {et }} \neq 0$ and $\dot{Q}^{\text {et }}=0$ (opposite to the LF case!). Thus the singular behavior of the NG-boson zero mode (3.7) (or (4.34)) may be understood as a remnant of the Lagrangian symmetry, an analogue of the NG theorem in the equal-time quantization.

The zero mode problem was also discussed in the continuum theory with careful treatment of the boundary condition. It was demonstrated that as far as the sign function is used for the commutator, the LF charge does not annihilate the vacuum in sharp contrast to DLCQ, since the zero mode as an accumulating point cannot be removed by simply dropping the exact zero mode with $p^{+} \equiv 0$ which is just measure zero. We also suggested that the " $\nu$ theory" might give a possible way out of this nontrivial vacuum problem in the continuum theory and give rise to the same result as that in DLCQ.

The non-conservation of the SSB charge on the LF was also stressed by Ida 26] and Carlitz et al. 28 long time ago in the continuum theory but not in DLCQ. Their way to define the LF charge is somewhat similar to ours, namely, the explicit mass of NG boson is kept finite in order to pick up the current matrix element with the NG-boson pole term dropped. However, they discussed it in the continuum theory without consistent treatment of the boundary condition and without realizing the zero mode problem. If they were careful enough about the boundary condition in the continuum theory, they would have arrived at 
difficulty of the nontrivial vacuum as we mentioned before. So it is essentially different from our argument in DLCQ.

Finally, we should mention that there is a more serious zero-mode problem in the continuum LF theory, namely the no-go theorem found by Nakanishi and Yamawaki [17]. The LF canonical commutator (5.10) gives explicit expression of two-point Wightman function on LF:

$$
\left.\langle 0|\phi(x) \phi(0)| 0\rangle\right|_{x^{+}=0}=\frac{1}{2 \pi} \int_{0}^{\infty} \frac{d p^{+}}{2 p^{+}} e^{-i p^{+} x^{-}} \cdot \delta^{(2)}\left(x^{\perp}\right) \quad,
$$

which is logarithmically divergent at $p^{+}=0$ and local in $x^{\perp}$ and, more importantly, is independent of the interaction and the mass. We can easily check this result in the free theory [29] where the theory is explicitly solved in all space-time and the two-point Wightman function is given at any point $x$ by the well-known invariant delta function $\Delta^{(+)}\left(x ; m^{2}\right)$ which is written in terms of the Hankel function $K_{1}$ in the space-like region $x^{2}<0$ :

$$
\begin{aligned}
\langle 0|\phi(x) \phi(0)| 0\rangle & =\Delta^{(+)}\left(x ; m^{2}\right)=\frac{1}{(2 \pi)^{3}} \int_{0}^{\infty} \frac{d p^{+}}{2 p^{+}} \int_{-\infty}^{\infty} d p^{\perp} e^{-i p^{-} x^{+}-i p^{+} x^{-}+i p^{\perp} x^{\perp}} \\
& =\frac{m}{4 \pi^{2} \sqrt{-x^{2}}} K_{1}\left(m \sqrt{-x^{2}}\right) \quad\left(x^{2}<0\right)
\end{aligned}
$$

Restricting (6.2) to the LF, $x^{+}=0$, yields

$$
\left.\langle 0|\phi(x) \phi(0)| 0\rangle\right|_{x^{+}=0}=\frac{m}{4 \pi^{2} \sqrt{x_{\perp}^{2}}} K_{1}\left(m \sqrt{x_{\perp}^{2}}\right)
$$

which is finite (positive definite), nonlocal in $x^{\perp}$ and dependent on mass, in obvious contradiction to the above result (6.1). Hence, already for the free field the LF quantization fails to reproduce the Lorentz-invariant theory. Actually, the latter Lorentz-invariant result (6.3) is a consequence of the mass-dependent regularization of $1 / p^{+}$singularity at $p^{+} \rightarrow 0$ by the infinitely oscillating (mass-dependent) phase factor $e^{-i p^{-} x^{+}}=e^{-i\left(m^{2}+p_{\perp}^{2}\right) / 2 p^{+} \cdot x^{+}}$in the integral of (6.2) before taking the LF restriction $x^{+}=0$. The LF quantization, restricting to $x^{+}=0$ beforehand, in fact kills such a regularizing factor and leads to a wrong result (6.1). Thus the LF restriction from the beginning loses all the information of dynamics carried by 
the zero mode as the accumulating point. This implies that even a free theory does not exist on the LF 17].

One might suspect that this conclusion could be an artifact of too formal argument and irrelevant to the actual physics, since one can construct free particle states, namely a free Fock space, with the correct spectra, as far as the momentum space consideration is concerned. However, the above result implies that quantum field on LF is ill-defined as the operator-valued distribution and so is the operator product on LF. Then it is rather difficult to construct a realistic LF Hamiltonian (with interaction) in terms of the products of local fields on the same $L F$ in a way consistent with the Lorentz invariance, which would be a serious problem even for practical physicists.

In fact, the above difficulty also applies to the interacting theory satisfying the Wightman axioms (no-go theorem) [17, in which case we have a spectral representation (UmezawaKamefuchi-Källen-Lehmann representation) for the commutator function:

$$
\begin{aligned}
\langle 0|[\phi(x), \phi(0)]| 0\rangle & =i \int_{0}^{\infty} d \mu^{2} \rho\left(\mu^{2}\right) \Delta\left(x ; \mu^{2}\right) \\
\int_{0}^{\infty} d \mu^{2} \rho\left(\mu^{2}\right) & =1, \quad \rho\left(\mu^{2}\right) \geq 0 .
\end{aligned}
$$

If one assumed that LF restriction $x^{+}=0$ of the theory were well-defined, then it would follow:

$$
\left.\langle 0|[\phi(x), \phi(0)]| 0\rangle\right|_{x^{+}=0}=\left.i \int_{0}^{\infty} d \mu^{2} \rho\left(\mu^{2}\right) \Delta\left(x ; \mu^{2}\right)\right|_{x^{+}=0}=-\frac{i}{4} \epsilon\left(x^{-}\right) \delta^{(2)}\left(x^{\perp}\right)
$$

since $\left.i \Delta\left(x ; \mu^{2}\right)\right|_{x^{+}=0}=-\frac{i}{4} \epsilon\left(x^{-}\right) \delta^{(2)}\left(x^{\perp}\right)$ is independent of $\mu^{2}$. Taking the $p^{+}>0$ part of the Fourier component of the commutator function (6.5), one would further obtain exactly the same result as (6.1) for the two-point Wightman function at $x^{+}=0$, this time $\phi$ being the interacting Heisenberg field instead of the free field. On the other hand, the same Wightman axioms yield the spectral representation also for the two-point Wightman function:

$$
\langle 0|\phi(x) \phi(0)| 0\rangle=\int_{0}^{\infty} d \mu^{2} \rho\left(\mu^{2}\right) \Delta^{(+)}\left(x ; \mu^{2}\right)
$$


Now, the LF restriction of (6.6) depends on $\rho\left(\mu^{2}\right)$ and does not agree with (6.1), since $\left.\Delta^{(+)}\left(x ; \mu^{2}\right)\right|_{x^{+}=0}$ given as (6.3) does depend on $\mu^{2}$, in sharp contrast to (6.1) which was derived from (6.5). Thus we have arrived at self-contradiction within the framework of Wightman axioms under the assumption that LF restriction is well-defined.

An immediate way to resolve this trouble would be to define the theory on the "near LF", $x^{+} \neq 0$, slightly away from the exact LF, $x^{+} \equiv 0$, and then take the LF limit $x^{+} \rightarrow 0$ only in the end of whole calculation as in (6.3). In fact such a prescription was first proposed by Nakanishi and Yabuki [29] in the continuum framework and later by Prokhvatilov et al. and others [30 in the context of DLCQ. However, it was noted [17 that the price to pay in this approach is non-vanishing vacuum polarization as in the equal-time quantization and hence we must give up the trivial vacuum, or physical Fock space, which is the most important feature of the LF quantization. Then there is no advantage of this approach over the equal-time quantization, concerning the simplicity of the vacuum in non-perturbative studies. Indeed, it was demonstrated more explicitly [31] that the vacuum is nontrivial and there exists nontrivial renormalization in the LF Hamiltonian in this approach: It is no longer simple to solve dynamics compared with the equal-time quantization.

Thus, in spite of its difficulties with the above no-go theorem, we must take the quantization on the exact LF, $x^{+} \equiv 0$, from the beginning in order to keep the trivial vacuum and physical Fock space. Actually, the no-go theorem implies that the LF restriction is not compatible with the Wightman axioms. Therefore, in order to make the theory well-defined on the exact LF, we are forced to give up some of the Wightman axioms, most naturally the Lorentz invariance. Indeed, DLCQ defined on the exact LF is such a theory: The theory itself explicitly violates the Lorentz invariance for $L<\infty$ and never recovers it even in the limit of $L \rightarrow \infty$ [17], as we shall see later. At the sacrifice of the Lorentz invariance, the trivial vacuum is in fact realized in DLCQ [5] as we have seen in this paper. The same is true in the $\nu$-theory [17] as we discussed in Section 5 and further demonstrate in the following. 
In the $\nu$-theory the two-point Wightman function for the free theory is given by [17:

$$
\begin{aligned}
\Delta_{\nu}^{(+)}\left(x ; m^{2}\right) & =\frac{c_{\nu}}{(2 \pi)^{3}} \int_{0}^{\infty} \frac{d p^{+}}{2 p^{+}}\left|p^{+}\right|^{\nu} \int_{-\infty}^{\infty} d p^{\perp} e^{-i p^{-} x^{+}-i p^{+} x^{-}+i p^{\perp} x^{\perp},} \\
& =\frac{c_{\nu}\left[e^{i \pi / 2}\left(x^{+}-i 0\right)\right]^{\nu}}{4 \pi^{2}}\left(\frac{m}{\rho}\right)^{1+\nu} K_{1+\nu}(m \rho) \\
c_{\nu}(=\text { const. }) & >0 \quad\left(c_{0}=1\right), \quad \rho=\left[-2\left(x^{+}-i 0\right)\left(x^{-}-i 0\right)+x_{\perp}^{2}\right]^{\frac{1}{2}},
\end{aligned}
$$

where the extra factor $c_{\nu}\left|p^{+}\right|^{\nu}$ is the regularization of the zero-mode singularity $1 / p^{+}$as was mentioned in Section 5. The previous non-commutativity between the integral of (6.2) and $x^{+} \rightarrow 0$ is now traded for that between $\nu \rightarrow 0$ and $x^{+} \rightarrow 0$. If we take $\nu \rightarrow 0$ first and then $x^{+} \rightarrow 0$, we can reproduce correct Lorentz-invariant result (6.3), which is the same as the procedure to take the "near LF" to the LF limit $x^{+} \rightarrow 0$ [29, 30, 31]. If, on the other hand, we take $x^{+} \rightarrow 0$ and then $\nu \rightarrow 0$, we arrive at the non-invariant answer (6.1) again. Thus the theory itself (operator, Fock space, etc.) violates Lorentz invariance and never reproduces a Lorentz-invariant field theory even in the limit $\nu \rightarrow 0$. Conversely, the $\nu$-theory is well-defined on the exact LF at the sacrifice of the Lorentz invariance (a part of Wightman axioms).

Now, the real problem is how to recover Lorentz invariance of the physical quantity (cnumber) like $S$ matrix which, unlike the Wightman function, has no reference to the fixed $L F$, even though the theory itself, defined on the fixed exact LF, has no Lorentz-invariant limit. Indeed, it was pointed out [17] that as far as the perturbation theory is concerned, the $S$ matrix coincides in the limit of $\nu \rightarrow 0$ with the conventional Feynman rule result which is Lorentz-invariant, with one notable exception, namely the vanishing vacuum polarization graph due to the modification of the zero-mode contribution. Note that $\nu \rightarrow 0$ is to be taken after whole calculation, since the $\nu$-theory is defined on the exact LF only for $\nu>0$ (no $\nu=0$ theory exists on the exact LF, as dictated by the no-go theorem). In fact, the Feynman propagator of the $\nu$-theory takes the form [17]:

$$
\Delta_{F, \nu}\left(x ; m^{2}\right)=\frac{i c_{\nu}}{(2 \pi)^{4}} \int d \vec{p}\left|p^{+}\right|^{\nu} \int d p^{-} \frac{e^{-i p^{-} x^{+}+i \vec{p} \vec{x}}}{2 p^{-} p^{+}-p_{\perp}^{2}-m^{2}+i 0}
$$


Then the vacuum polarization graph calculated by the standard LF method 32 does vanish 17]:

$$
\begin{aligned}
\int d p^{+}\left|p^{+}\right|^{\nu} \int d p^{-} \frac{F\left(p^{+} p^{-}\right)}{2 p^{+} p^{-}-m^{2}+i 0} & =\int_{-\infty}^{\infty} d \lambda \tilde{F}(\lambda) \int_{0}^{\infty} d \xi \frac{e^{-i m^{2} \xi}}{i} \int d p^{+}\left|p^{+}\right|^{\nu} \int d p^{-} e^{i 2 p^{+} p^{-}(\xi+\lambda)} \\
& =C \int d p^{+}\left|p^{+}\right|^{\nu} \delta\left(p^{+}\right)=0
\end{aligned}
$$

where $F$ and $\tilde{F}$ are a certain function and its Fourier transform with respect to $\lambda$, respectively and $C=-\pi i \int d \lambda d \xi e^{-i m^{2} \xi} \tilde{F}(\lambda) /(\lambda+\xi)$ a numerical constant, and we have disregarded the transverse part which is irrelevant. Note that the zero-mode contribution $\delta\left(p^{+}\right)$has been modified by the extra factor $\left|p^{+}\right|^{\nu}(\nu>0)$ so as to yield zero vacuum polarization. This is consistent with the previous argument in Section 5 on the trivial vacuum $Q|0\rangle=0$ in the $\nu$-theory. In contrast, all other graphs having no $\delta\left(p^{+}\right)$would be unaffected by the extra factor $\left|p^{+}\right|^{\nu}$ and thus reproduce the usual Lorentz-invariant result in the $\nu \rightarrow 0$ limit. The vanishing vacuum polarization (6.9) is in sharp contrast to the case where we take $\nu \rightarrow 0$ beforehand (no $\left|p^{+}\right|^{\nu}$ factor), which actually corresponds to the prescription [29, 30, 31] approaching from "near LF" to LF, with $\nu=0$ : In such a case we have a non-vanishing vacuum polarization as in the equal-time quantization, the whole contribution coming from the zero mode $\int d p^{+} \delta\left(p^{+}\right) \neq 0$, as was noted by Chang and Ma 32.

We can also expect the same situation in DLCQ. The theory itself is not Lorentz-invariant, since the two-point Wightman function in the free theory takes the form:

$$
\begin{aligned}
\left.\Delta_{\mathrm{DLCQ}}^{(+)}\left(x ; m^{2}\right)\right|_{x^{+}=0} & =\frac{1}{2 \pi} \sum_{n>0} \frac{\pi}{L} \frac{1}{2 p_{n}^{+}} e^{-i p_{n}^{+} x^{-}} \cdot \delta^{(2)}\left(x^{\perp}\right) \\
p_{n}^{+} & =\frac{n \pi}{L} \quad(n=1,2, \cdots)
\end{aligned}
$$

which coincides with (6.1) in the continuum limit of $L \rightarrow \infty$ (with $p_{n}^{+}=n \pi / L=$ fixed), again in disagreement with the Lorentz invariance [17]. Note that the sum does not include the zero mode $n=0$, since the zero mode in the free theory vanishes through the zero-mode constraint (2.26). Although the theory itself is not Lorentz-invariant, we would reproduce the 
Lorentz-invariant result for the $S$ matrix except for the vacuum polarization in the continuum limit of $L \rightarrow \infty$, as far as the perturbation theory is concerned. In fact, Feynman propagator in DLCQ takes the form:

$$
\Delta_{F, \mathrm{DLCQ}}\left(x ; m^{2}\right)=\frac{i}{(2 \pi)^{4}} \sum_{n= \pm 1, \pm 2, \cdots} \frac{\pi}{L} \int d p^{\perp} d p^{-} \frac{e^{-i p^{-} x^{+}-i p_{n}^{+} x^{-}+i p^{\perp} x^{\perp}}}{2 p^{-} p_{n}^{+}-p_{\perp}^{2}-m^{2}+i 0}
$$

where again the zero mode $n=0$ is not included in the sum. When this is used in the Feynman rule for the perturbation, the absence of the zero mode $n=0$ actually dictates that vacuum polarization graph does vanish similarly to (6.9):

$$
\sum_{n= \pm 1, \pm 2, \cdots} \frac{\pi}{L} \int d p^{-} \frac{F\left(p^{+} p^{-}\right)}{2 p^{+} p^{-}-m^{2}+i 0}=C \sum_{n= \pm 1, \pm 2, \cdots} \frac{\pi}{L} \delta\left(p_{n}^{+}\right)=0
$$

which is consistent with the trivial vacuum already established [5] through the zero-mode constraint (see Section 2). Note that the continuum limit $(\mathrm{E} \rightarrow \infty)$ of (6.12) obviously disagree with the covariant result [32] $C \int d p^{+} \delta\left(p^{+}\right) \quad(\neq 0)$. In contrast, all other graphs having no $\delta\left(p_{n}^{+}\right)$are insensitive to the zero mode $n=0$ and hence would coincide with the covariant result in such a limit.

To summarize, the no-go theorem forbids the well-defined LF restriction of the Lorentzinvariant field theory due to the peculiarity of the zero mode as an accumulating point in the continuum framework. Conversely, the theory defined on the exact LF such as DLCQ or $\nu$-theory, although realizing the trivial vacuum and no vacuum polarization, would never recover the Lorentz-invariance even in the limit of $L \rightarrow \infty$ or $\nu \rightarrow 0$. Thus the Lorentzinvariant limit in such a theory can only be realized on the c-number physical quantity like S matrix which has no reference to the fixed LF but not on the theory itself (Fock space, operator, etc). In fact, we have discussed that as far as the perturbation theory is concerned, both DLCQ and $\nu$-theory would reproduce the Lorentz-invariant S matrix, while keeping the vacuum polarization absent (no zero-mode contribution) in accordance with the trivial vacuum. This was shown through the explicit solution of the perturbative dynamics which is based on the interaction picture with the propagator being given by the free theory 
whose solution is completely known not only on a fixed $\mathrm{LF} x^{+}=0$ but also on other region $x^{+} \neq 0$.

However, the real purpose of the LF quantization is to solve the dynamics non-perturbatively in a way much simpler than the equal-time quantization, based on the trivial vacuum and the physical Fock space for the interacting Heisenberg field. Then, in order to reproduce the Lorentz invariance without recourse to the perturbation theory, we actually would need explicit solution of the non-perturbative dynamics itself, particularly the zero mode solution. Thus, recovering the Lorentz invariance is a highly dynamical issue in the LF quantization, the situation being somewhat analogous to the lattice gauge theories. Then it remains a big challenge for the LF quantization to overcome the no-go theorem in the non-perturbative way. Particularly in DLCQ we would need to find the non-perturbative solution to the zeromode constraint which might play a crucial role in taking the continuum limit $L \rightarrow \infty$ so as to recover the Lorentz invariance in the physical quantity (c-number).

In this paper we did not attempt to solve the above no-go theorem in the non-perturbative sense. Instead, in DLCQ (or in the $\nu$-theory), we only made a rather modest attempt to solve the easier one, namely, to formulate the SSB in a manner consistent with the trivial LF vacuum. No doubt, fully non-perturbative solution to the no-go theorem is highly desired. Much work is to be done towards revealing the non-perturbative structure of the LF theory through the zero mode.

\section{Acknowledgments}

We would like to thank K.G. Wilson for fruitful discussion. We also appreciate stimulating discussion with M. Taniguchi. S.T. is supported by Max-Planck Gesellschaft Stipendium and thanks H.C. Pauli for encouragement and his interest in this work. K.Y. is supported in part by the Grant-in-Aid of the Japanese Ministry of Education, Science and Culture (\# 08640365) and the Sumitomo Science Foundation. 


\section{A DLCQ Canonical Commutator}

Here we give a detailed derivation of the DLCQ canonical commutator (2.29).

There are three constraints (2.9), (2.10) and (2.19):

$$
\begin{aligned}
\Phi_{1}(x) & \equiv \pi_{\varphi}(x)-\partial_{-} \varphi(x) \approx 0 \\
\Phi_{2}(x) & \equiv \pi_{0}(x) \approx 0 \\
\Phi_{3}(x) & \equiv \frac{1}{2 L} \int_{-L}^{L} d x^{-}\left[\left(\mu^{2}-\partial_{\perp}^{2}\right) \phi+\frac{\partial V}{\partial \phi}\right] \approx 0 .
\end{aligned}
$$

Using (2.12) - (2.14), we compute $C_{i, j}(x, y) \equiv\left\{\Phi_{i}(x), \Phi_{j}(y)\right\}$, whose non-zero matrix elements are given by

$$
\begin{aligned}
& C_{1,1}(x, y)=\left(\partial_{-}^{y}-\partial_{-}^{x}\right) \delta^{(3)}(\vec{x}-\vec{y}), \\
& C_{1,3}(x, y)=-\frac{1}{2 L}\left\{\alpha(\vec{x})-\frac{1}{2 L} \beta^{-1}\left(x^{\perp}\right)\right\} \delta^{(2)}\left(x^{\perp}-y^{\perp}\right)=-C_{3,1}(y, x), \\
& C_{2,3}(x, y)=-\frac{1}{4 L^{2}} \beta^{-1}\left(x^{\perp}\right) \delta^{(2)}\left(x^{\perp}-y^{\perp}\right)=-C_{3,2}(y, x),
\end{aligned}
$$

where $x^{+}=y^{+}$is understood and $\alpha(\vec{x})$ and $\beta^{-1}\left(x^{\perp}\right)$ are given in (2.30). Note that $\frac{1}{2 L} \beta^{-1}$ is the zero mode of $\alpha$.

Let us now calculate the inverse matrix $M_{i, j}(x, y) \equiv\left(C^{-1}\right)_{i, j}(x, y)$, which is the essential step to obtain the Dirac bracket (2.22). It is easy to see that non-zero matrix elements of $M_{i, j}$ are given by

$$
\begin{aligned}
M_{1,1}(x, y)=\left(C_{1,1}\right)^{-1}(x, y) & =-\frac{1}{4}\left[\epsilon\left(x^{-}-y^{-}\right)-\frac{x^{-}-y^{-}}{L}\right] \cdot \delta^{(2)}\left(x^{\perp}-y^{\perp}\right) \\
M_{1,2}(x, y) & \equiv p\left(\vec{x}, y^{\perp}\right) \\
M_{2,1}(x, y) & \equiv q\left(x^{\perp}, \vec{y}\right) \\
M_{2,2}(x, y) & \equiv r\left(x^{\perp}, y^{\perp}\right) \\
M_{2,3}(x, y)=\left(C_{3,2}\right)^{-1}(x, y) & =4 L^{2} \beta\left(y^{\perp}\right) \cdot \delta^{(2)}\left(x^{\perp}-y^{\perp}\right)=-M_{3,2}(y, x)
\end{aligned}
$$

where the functions $p, q$ and $r$ satisfy the equations:

$$
\int d \vec{y} C_{1,1}(x, y) p\left(\vec{y}, z^{\perp}\right)+\int d y^{\perp} C_{1,3}(x, y) M_{3,2}(y, z)=0
$$




$$
\begin{aligned}
& \int d \vec{y} C_{3,1}(x, y) M_{1,1}(y, z)+\int d y^{\perp} C_{3,2}(x, y) q\left(y^{\perp}, \vec{z}\right)=0 \\
& \int d \vec{y} C_{3,1}(x, y) p\left(\vec{y}, z^{\perp}\right)+\int d y^{\perp} C_{3,2}(x, y) r\left(y^{\perp}, z^{\perp}\right)=0
\end{aligned}
$$

We readily find the solutions:

$$
\begin{aligned}
p\left(\vec{y}, z^{\perp}\right)= & -\int d \vec{w}_{1} d w_{2}^{\perp} M_{1,1}\left(y, w_{1}\right) C_{1,3}\left(w_{1}, w_{2}\right) M_{3,2}\left(w_{2}, z\right) \\
= & \frac{L}{2} \int_{-L}^{L} d u^{-}\left[\epsilon\left(y^{-}-u^{-}\right)-\frac{y^{-}-u^{-}}{L}\right] \beta\left(z^{\perp}\right) \alpha\left(u^{-}, z^{\perp}\right) \delta^{(2)}\left(y^{\perp}-z^{\perp}\right) \quad, \quad(\mathrm{A} .15) \\
q\left(y^{\perp}, \vec{z}\right)= & -\int d w_{1}^{\perp} d \vec{w}_{2} M_{2,3}\left(y, w_{1}\right) C_{3,1}\left(w_{1}, w_{2}\right) M_{1,1}\left(w_{2}, z\right) \\
= & \frac{L}{2} \int_{-L}^{L} d u^{-} \beta\left(y^{\perp}\right) \alpha\left(u^{-}, y^{\perp}\right)\left[\epsilon\left(u^{-}-z^{-}\right)-\frac{u^{-}-z^{-}}{L}\right] \delta^{(2)}\left(y^{\perp}-z^{\perp}\right) \\
= & -p\left(\vec{z}, y^{\perp}\right), \\
r\left(y^{\perp}, z^{\perp}\right)= & \int d w_{1}^{\perp} d \vec{w}_{2} d \vec{w}_{3} d w_{4}^{\perp} M_{2,3}\left(y, w_{1}\right) C_{3,1}\left(w_{1}, w_{2}\right) M_{1,1}\left(w_{2}, w_{3}\right) \\
& \times C_{1,3}\left(w_{3}, w_{4}\right) M_{3,2}\left(w_{4}, z\right) \\
= & -L^{2} \int d w_{2}^{-} d w_{3}^{-} \beta\left(y^{\perp}\right) \alpha\left(w_{2}^{-}, y^{\perp}\right)\left[\epsilon\left(w_{2}^{-}-w_{3}^{-}\right)-\frac{w_{2}^{-}-w_{3}^{-}}{L}\right] \beta\left(z^{\perp}\right) \alpha\left(w_{3}^{-}, z^{\perp}\right) \\
& \times \delta^{(2)}\left(y^{\perp}-z^{\perp}\right),
\end{aligned}
$$

where we have used

$$
\int_{-L}^{L} d u^{-}\left[\epsilon\left(u^{-}-v^{-}\right)-\frac{u^{-}-v^{-}}{L}\right]=\int_{-L}^{L} d v^{-}\left[\epsilon\left(u^{-}-v^{-}\right)-\frac{u^{-}-v^{-}}{L}\right]=0
$$

Now we are ready to derive the Dirac bracket for the full field $\phi=\varphi+\phi_{0}$ :

$$
\begin{aligned}
\{\phi(\vec{x}), \phi(\vec{y})\}_{D B}= & \{\varphi(\vec{x}), \varphi(\vec{y})\}_{D B}+\left\{\phi_{0}\left(x^{\perp}\right), \varphi(\vec{y})\right\}_{D B}+\left\{\varphi(\vec{x}), \phi_{0}\left(y^{\perp}\right)\right\}_{D B} \\
& +\left\{\phi_{0}\left(x^{\perp}\right), \phi_{0}\left(y^{\perp}\right)\right\}_{D B}
\end{aligned}
$$

According to (2.22), each Dirac bracket is evaluated as follows:

$$
\begin{aligned}
\{\varphi(\vec{x}), \varphi(\vec{y})\}_{D B} & =M_{1,1}(x, y)=-\frac{1}{4}\left[\epsilon\left(x^{-}-y^{-}\right)-\frac{x^{-}-y^{-}}{L}\right] \delta^{(2)}\left(x^{\perp}-y^{\perp}\right) \\
\left\{\varphi(\vec{x}), \phi_{0}\left(y^{\perp}\right)\right\}_{D B} & =\frac{1}{2 L} \cdot p\left(\vec{x}, y^{\perp}\right)
\end{aligned}
$$




$$
\begin{aligned}
= & \frac{1}{4} \int_{-L}^{L} d u^{-}\left[\epsilon\left(x^{-}-u^{-}\right)-\frac{x^{-}-u^{-}}{L}\right] \beta\left(y^{\perp}\right) \alpha\left(u^{-}, y^{\perp}\right) \\
& \times \delta^{(2)}\left(x^{\perp}-y^{\perp}\right) \\
\left\{\phi_{0}\left(x^{\perp}\right), \varphi(\vec{y})\right\}_{D B}= & \frac{1}{2 L} \cdot q\left(x^{\perp}, \vec{y}\right)=-\left\{\varphi(\vec{y}), \phi_{0}\left(x^{\perp}\right)\right\}_{D B} \\
= & \frac{1}{4} \int_{-L}^{L} d u^{-} \beta\left(x^{\perp}\right) \alpha\left(u^{-}, x^{\perp}\right)\left[\epsilon\left(u^{-}-y^{-}\right)-\frac{u^{-}-y^{-}}{L}\right] \\
\left\{\phi_{0}\left(x^{\perp}\right), \phi_{0}\left(y^{\perp}\right)\right\}_{D B}= & \frac{1}{4 L^{2}} \cdot r\left(x^{\perp}, y^{\perp}\right) \\
= & -\frac{1}{4} \int d u^{-} d v^{-} \beta\left(x^{\perp}\right) \alpha\left(u^{-}, x^{\perp}\right)\left[\epsilon\left(u^{-}-v^{-}\right)-\frac{u^{-}-v^{-}}{L}\right] \beta\left(y^{\perp}\right) \alpha\left(v^{-}, y^{\perp}\right) \\
& \times \delta^{(2)}\left(x^{\perp}-y^{\perp}\right),
\end{aligned}
$$

where use has been made of (A.15)-(A.17) and the Poisson brackets (2.12)-(2.14).

Combining (A.20)-(A.23) into (A.19) and making replacement $i\{A, B\}_{D B} \rightarrow[A, B]$, we arrive at the DLCQ canonical commutator (2.29):

$$
\begin{aligned}
{[\phi(x), \phi(y)]=} & -\frac{i}{4} \int d u^{-} d v^{-}\left[\beta\left(x^{\perp}\right) \alpha\left(u^{-}, x^{\perp}\right)-\delta\left(u^{-}-x^{-}\right)\right]\left[\epsilon\left(u^{-}-v^{-}\right)-\frac{u^{-}-v^{-}}{L}\right] \\
& \times\left[\beta\left(y^{\perp}\right) \alpha\left(v^{-}, y^{\perp}\right)-\delta\left(v^{-}-y^{-}\right)\right] \delta^{(2)}\left(x^{\perp}-y^{\perp}\right)
\end{aligned}
$$

Next we demonstrate that the above commutator (2.29) actually coincides with (2.31), the one obtained in Ref.[5], up to operator ordering, i.e., in the sense of Dirac bracket. First note that (A.23) is actually vanishing and so is the zero-mode-zero-mode commutator $\left[\phi_{0}(x), \phi_{0}(y)\right]:$

$$
r\left(x^{\perp}, y^{\perp}\right)=0
$$

where use has been made of (A.18) and

$$
\int d u^{-} \int d v^{-}\left[\epsilon\left(u^{-}-v^{-}\right)-\frac{u^{-}-v^{-}}{L}\right] \alpha\left(u^{-}, x^{\perp}\right) \alpha\left(v^{-}, y^{\perp}\right) \delta^{(2)}\left(x^{\perp}-y^{\perp}\right)=0
$$

Eq.(A.25) also follows from the antisymmetric property of $M_{i, j}(x, y)$ and the one-dimensionality of $M_{2,2}$ in $x^{-}\left(y^{-}\right)$direction: i,e, $M_{2,2}(x, y)=r\left(x^{\perp}, y^{\perp}\right)=-M_{2,2}(y, x)=0 \quad$. 
The sum of (A.21) and (A.22) can be rewritten as

$$
\begin{aligned}
& \frac{1}{4} \int d u^{-} \beta\left(x^{\perp}\right) \alpha\left(u^{-}, x^{\perp}\right)\left[\epsilon\left(u^{-}-y^{-}\right)-\frac{u^{-}-y^{-}}{L}\right] \delta^{(2)}\left(x^{\perp}-y^{\perp}\right) \\
& +\frac{1}{4} \int d u^{-}\left[\epsilon\left(x^{-}-u^{-}\right)-\frac{x^{-}-u^{-}}{L}\right] \beta\left(y^{\perp}\right) \alpha\left(u^{-}, y^{\perp}\right) \delta^{(2)}\left(x^{\perp}-y^{\perp}\right) \\
= & \frac{1}{4} \int d u^{-}\left[\epsilon\left(u^{-}-y^{-}\right)+\epsilon\left(x^{-}-u^{-}\right)\right] \beta \alpha\left(u^{-}\right) \delta^{(2)}\left(x^{\perp}-y^{\perp}\right) \\
& -\frac{1}{4} \int d u^{-}\left[\frac{u^{-}-y^{-}}{L}+\frac{x^{-}-u^{-}}{L}\right] \beta \alpha\left(u^{-}\right) \delta^{(2)}\left(x^{\perp}-y^{\perp}\right) \\
= & \frac{1}{2} \int_{y^{-}}^{x^{-}} d u^{-} \beta \alpha\left(u^{-}\right) \delta^{(2)}\left(x^{\perp}-y^{\perp}\right)-\frac{1}{4}\left[\frac{x^{-}-y^{-}}{L}\right] \delta^{(2)}\left(x^{\perp}-y^{\perp}\right),
\end{aligned}
$$

which is combined with (A.20), yielding

$$
\{\phi(x), \phi(y)\}_{D B}=-\frac{1}{4}\left[\epsilon\left(x^{-}-y^{-}\right)-2 \beta \int_{y^{-}}^{x^{-}} d z^{-} \alpha\left(z^{-}\right)\right] \cdot \delta^{(2)}\left(x^{\perp}-y^{\perp}\right) .
$$

Upon replacement $i\{A, B\}_{D B} \rightarrow[A, B]$, we finally obtain (2.31).

\section{B The Problem of Boundary Condition}

We usually assign the boundary condition in DLCQ, because the "space"coordinates are confined in the finite box $-L \leq x^{-} \leq L$. Besides such a practical reason, there is an inevitable reason why the boundary condition on $x^{-}$direction must be specified. This is not a specific problem of DLCQ, but a common problem to the whole LF quantization including the "continuum" framework. To emphasize that the consistent LF quantization must be accompanied by the boundary condition we reexamine the special role of the boundary condition according to Steinhardt [18] and study what kind of boundary condition is consistent in DLCQ.

Let us consider the "continuum" or "discretized" LF quantization of scalar model without boundary condition in the context of the Dirac quantization in Sect.2. Due to no boundary condition, the constraint for zero mode will not appear. The only constraint appearing in 
the theory is

$$
\Phi(x)=\pi(x)-\partial_{-} \phi(x),
$$

whose Poisson bracket is given by

$$
\{\Phi(x), \Phi(y)\}=\left(\partial_{-}^{y}-\partial_{-}^{x}\right) \delta^{(3)}(\vec{x}-\vec{y}) \quad .
$$

Strictly speaking, we have infinitely many constraints which are expressible as linear combination of (B.1).

An important observation in [18] is that there is a subset of constraints which appears to be not only first class but also second class. To see this, consider a linear combination of the primary constraint

$$
\Phi_{0} \equiv \int d x^{-} \Phi(x),
$$

which corresponds to the "zero mode" of $\Phi(x)$ in the discretized theory. Suppose that any surface term is neglected throughout the calculation, one can easily find

$$
\left\{\Phi_{0}, \Phi(x)\right\}=0 \quad .
$$

This means that $\Phi_{0}$ is first class, because it should commute with any linear combination of $\Phi(x)$ as a consistency. However, this is not always the case, as the following example is illustrating:

$$
\begin{aligned}
\left\{\Phi_{0}, \int \epsilon\left(y^{-}\right) \Phi(y) d y^{-}\right\} & =-2 \int d x^{-} d y^{-} \partial_{-}^{y} \epsilon\left(y^{-}\right) \delta^{(3)}(\vec{x}-\vec{y}) \\
& =-4 \delta^{(2)}\left(x^{\perp}-y^{\perp}\right) \neq 0,
\end{aligned}
$$

where $\epsilon(x)$ is the sign function. This means that $\Phi_{0}$ is second class in contradiction with the previous result. Actually, $\Phi_{0}$ is neither first class nor second class, which represents inconsistency hidden in the theory. This ambiguity reveals itself as the ambiguity of the inverse matrix of constraints, $C^{-1}$ in (2.22), and that of the Lagrange multiplier $v(x)$. It is easily shown that all such ambiguities can be removed, once the boundary condition at $x^{-}= \pm \infty$ or $x^{-}= \pm L$ is specified. 
Let us then study the possible boundary conditions in DLCQ. Although the same problem was studied by Steinhardt, he discussed it within the continuum framework and neglected all surface terms appearing in the partial integrations. So we study the same problem by carefully treating surface terms in DLCQ. For this purpose, we generalize $\Phi_{0}$ and consider the following constraint which appears in the total Hamiltonian:

$$
\Phi[v]=\int_{-L}^{L} d x^{-} v(x) \Phi(x)
$$

where $v(x)$ is a certain function (Lagrange multiplier) which satisfies the same boundary condition as $\phi(x)$ [5]. Once the boundary condition is specified, providing $\Phi[v]$ for all $v$ becomes equivalent to providing $\Phi(x)$ for all $x$, which is nothing but the necessary condition for consistency mentioned above. Moreover, we demand that the variation of canonical variable generated by (B.6) must satisfy the same boundary condition. We can derive this condition by writing down the functional variation of $\Phi[v]$ :

$$
\begin{aligned}
\delta \Phi[v] & =\int_{-L}^{L} d x^{-}\left[v(x) \delta \pi(x)+\partial_{-} v(x) \delta \phi(x)\right] \\
& -v\left(x^{-}=L\right) \delta \phi\left(x^{-}=L\right)+v\left(x^{-}=-L\right) \delta \phi\left(x^{-}=-L\right),
\end{aligned}
$$

where the first two terms on the r.h.s. give the canonical variation of the fields which preserve the same boundary condition as the canonical variables. On the other hand, the surface terms generally violate the boundary condition. One can thus require the condition

$$
v\left(x^{-}=L\right) \delta \phi\left(x^{-}=L\right)=v\left(x^{-}=-L\right) \delta \phi\left(x^{-}=-L\right),
$$

which is nothing but the discretized version of that derived in [18]. This includes the periodic boundary condition studied in Ref. [5].

Based on this condition we investigate what kind of boundary condition can exist consistently. We pick up here some typical ones other than periodic case;

(I) the first boundary value: $\phi\left(x^{-}=L\right)=\phi\left(x^{-}=-L\right)=0$,

(II) the second boundary value: $\frac{d}{d x^{-}} \phi\left(x^{-}=L\right)=\frac{d}{d x^{-}} \phi\left(x^{-}=-L\right)=0$, 
(III) the third boundary value: the mixed type of the above two conditions,

(IV) the anti-periodic boundary condition,

where the right hand sides of both (I) and (II) can be generalized to any value. Note that $\delta \phi(x)$ and $v(x)$ obey the same boundary condition as $\phi(x)$. Now, in the boundary condition (II) and (III) $\phi$ is left arbitrary at $x^{-}= \pm L$ and so are $\delta \phi(x)$ and $v(x)$, and hence the boundary condition (II) and (III) do not generally satisfy the condition (B.8).

Let us next consider the case (I), in which case it is helpful to use mode expansion:

$$
\phi(x)=\sum_{n=1}^{\infty} a_{n}\left(x^{+}, x^{\perp}\right) \sin \left\{\frac{n \pi}{2 L}\left(x^{-}+L\right)\right\} .
$$

The delta function should be interpreted as

$$
\delta\left(x^{-}-y^{-}\right)=\frac{1}{L} \sum_{n=1}^{\infty} \sin \left\{\frac{n \pi}{2 L}\left(x^{-}+L\right)\right\} \sin \left\{\frac{n \pi}{2 L}\left(y^{-}+L\right)\right\}
$$

It is shown that the inverse of the Dirac matrix, $C^{-1}(x, y)$, satisfying the condition,

$$
C^{-1}(x, y)=-C^{-1}(y, x), \quad C^{-1}(L, y)=-C^{-1}(-L, y)=0
$$

does not exist. Therefore the only constraint which may give the consistent theory is the case of the anti-periodic boundary condition.

\section{Anti-periodic boundary condition [33]}

The scalar field with the anti-periodic boundary condition is expanded by the complete sets $\left\{\frac{1}{\sqrt{2 L}} e^{-\frac{i n \pi}{L} x^{-}}\right\}_{n= \pm \frac{1}{2}, \pm \frac{3}{2} \ldots}$, where there is no zero mode due to the anti-periodic boundary condition. For the Lagrangian (2.3), the only constraint is the primary constraint $\Phi(x)=$ $\pi(x)-\partial_{-} \phi(x)$ which is second class. It is easily confirmed that the Poisson bracket

$$
\{\Phi(x), \Phi(y)\}=\left(-\partial_{-}^{x}+\partial_{-}^{y}\right) \delta\left(x^{-}-y^{-}\right) \delta^{(2)}\left(x^{\perp}-y^{\perp}\right)
$$

has its inverse

$$
\{\Phi(x), \Phi(y)\}^{-1}=-\frac{1}{4} \epsilon\left(x^{-}-y^{-}\right) \delta^{(2)}\left(x^{\perp}-y^{\perp}\right)
$$


in the meaning of the delta function

$$
\delta\left(x^{-}\right)=\sum_{n} \frac{1}{2 L} e^{-\frac{i n \pi}{L} x^{-}} \quad\left(n= \pm \frac{1}{2}, \pm \frac{3}{2} \cdots\right)
$$

Then the commutation relation is given by

$$
[\phi(x), \phi(y)]=-\frac{i}{4} \epsilon\left(x^{-}-y^{-}\right) \delta^{(2)}\left(x^{\perp}-y^{\perp}\right)
$$

where both sides of the equation show the consistent behaviors at $x^{-}= \pm L$.

Next we check the Poincaré invariance of the theory. In the case of periodic boundary condition it is shown that the Poincaré invariance is not recovered at least in the naive limit of $L \rightarrow \infty$ [5]. Hence it is interesting to study the same problem for anti-periodic boundary condition.

Let us first derive the equation of motion. The total Hamiltonian is described by

$$
H_{T}=\int d^{3} \vec{x}\left[\frac{1}{2}\left\{\left(\partial_{\perp} \phi\right)^{2}+\mu^{2} \phi^{2}\right\}+V(\phi)\right]+\int d^{3} \vec{x} v(x) \Phi(x)
$$

where $v$ is the Lagrange multiplier.

The consistency condition for the primary constraint $\Phi(x)$ reads

$$
\begin{aligned}
-2 \partial_{-} v & =\left(\mu^{2}-\partial_{\perp}^{2}\right) \phi+\frac{\partial V}{\partial \phi} \\
& \equiv \chi
\end{aligned}
$$

where the surface term for $v(x)$ is dropped by the boundary condition. Combined with

$$
\partial_{+} \phi(x)=\left\{\phi(x), H_{T}\right\}=v(x)
$$

we obtain

$$
2 \partial_{+} \partial_{-} \phi \approx-\chi
$$

By using this relation it is shown that

$$
\begin{aligned}
{\left[\phi(x), P^{-}\right] } & =\int_{-L}^{L} d^{3} y\left\{\left(\mu^{2}-\partial_{\perp}^{2}\right) \phi(y)+\frac{\partial V}{\partial \phi}\right\}[\phi(x), \phi(y)] \\
& =i \partial_{+} \phi(x)+\frac{i}{2}\left\{\partial_{+} \phi\left(x^{-}=L\right) \epsilon\left(x^{-}-L\right)-\partial_{+} \phi\left(x^{-}=-L\right) \epsilon\left(x^{-}+L\right)\right\} \\
& =i \partial_{+} \phi(x),
\end{aligned}
$$


where

$$
P^{-}=\int_{-L}^{L} d^{3} x\left[\frac{1}{2}\left\{\left(\partial_{\perp} \phi\right)^{2}+\mu^{2} \phi^{2}\right\}+V(\phi)\right]
$$

Similar arguments can be applied to other Poincaré generators:

$$
\begin{gathered}
{\left[\phi(x), P^{\mu}\right]=i \partial^{\mu} \phi(x),} \\
{\left[\phi(x), M^{i j}\right]=i\left(x^{i} \partial^{j}-x^{j} \partial^{i}\right) \phi(x),} \\
{\left[\phi(x), M^{i+}\right]=i\left(x^{i} \partial^{+}-x^{+} \partial^{i}\right) \phi(x),} \\
{\left[\phi(x), M^{+-}\right]=i\left(x^{+} \partial^{-}-x^{-} \partial^{+}\right) \phi(x),}
\end{gathered}
$$

which is compared with the periodic case [5] where (B.23) does not hold due to the zero mode. On the other hand, we have

$$
\begin{aligned}
{\left[\phi(x), M^{-i}\right] } & =i\left(x^{-} \partial^{i}-x^{i} \partial^{-}\right) \phi(x) \\
& -\frac{i}{4}\left[y^{-} \epsilon\left(x^{-}-y^{-}\right) \partial_{i} \phi(y)\right]_{y^{-}=-L}^{y^{-}=L}
\end{aligned}
$$

which is dependent on the box size $L$ and hence violates the Lorentz invariance even in the infinite volume limit as in the case of the periodic boundary condition [5]. Thus the Poincaré invariance does not hold with respect to $M^{-i}$. We need to devise an appropriate continuum limit instead of the naive limit to recover the Poincaré invariance of the theory. 


\section{Unbroken Phase of O(2)-Linear Sigma Model}

We describe the unbroken phase of the $\mathrm{O}(2)$-linear sigma model by treating the zero modes explicitly. In this phase we can explicitly check validity of our operator ordering, namely, the Weyl ordering used for studying the broken (SSB) phase of the sigma model in Sect.4.

For simplicity, let us consider the bosonic part (4.2) with $\mu^{2}>0$ and $c \equiv 0$. To solve the zero modes, it is convenient to divide them into the classical and the operator parts as done in the Section 4. The solution of the zero-mode constraints for the classical part is trivial, i.e. $v_{\pi}=v_{\sigma}=0$, implying that the classical contribution to the vacuum expectation value is zero. On the other hand, the operator part of the zero-mode constraints is still too complicated to solve nonperturvatively. Then we solve them perturbatively again. The lowest order solutions of the operator part of the zero modes are derived as

$$
\begin{aligned}
& \omega_{\pi}=\frac{\lambda}{2 L}\left(-\mu^{2}+\partial_{\perp}^{2}\right)^{-1} \int_{-L}^{L} d y^{-}\left(\varphi_{\pi}^{3}+\varphi_{\pi} \varphi_{\sigma}^{2}\right), \\
& \omega_{\sigma}=\frac{\lambda}{2 L}\left(-\mu^{2}+\partial_{\perp}^{2}\right)^{-1} \int_{-L}^{L} d y^{-}\left(\varphi_{\sigma}^{3}+\varphi_{\sigma} \varphi_{\pi}^{2}\right),
\end{aligned}
$$

both of which include no divergence and thus well-defined. These explicit solutions confirm the well-known properties in the unbroken phase.

First of all, the same transformation law as that in the equal-time quantization

$$
[Q, \pi]=i \sigma \quad, \quad[Q, \sigma]=-i \pi \quad
$$

follows from the direct calculation

$$
\left[Q, \omega_{\pi}\right]=i \omega_{\sigma} \quad, \quad\left[Q, \omega_{\sigma}\right]=-i \omega_{\pi}
$$

where

$$
Q=\int d^{3} \vec{x}\left(\partial_{-} \varphi_{\sigma} \varphi_{\pi}-\partial_{-} \varphi_{\pi} \varphi_{\sigma}\right)
$$

Second, (C.3) is consistent to the trivial property of the LF vacuum. Actually $\left\langle\omega_{\pi}\right\rangle=$ $\left\langle\omega_{\sigma}\right\rangle=0$ in addition to $\left\langle\varphi_{\pi}\right\rangle=\left\langle\varphi_{\sigma}\right\rangle=0$ lead to

$$
\langle[Q, \pi]\rangle=\langle[Q, \sigma]\rangle=0
$$


which is consistent with $Q|0\rangle=0$.

Now, let us check the operator ordering by checking the charge conservation which should hold in the unbroken phase in contrast to the broken phase. In fact an arbitrary operator ordering would not necessarily lead to the charge conservation due to the existence of zero modes. One should find such an operator ordering as to satisfy the charge conservation and the Weyl ordering actually does it. Indeed it is easy to show

$$
i \frac{d Q}{d x^{+}}=[Q, H]=0
$$

under the Weyl ordering. It is this property that the Weyl ordering was assumed even in the case of the broken phase in Sect.4. Although only the lowest order solution was explicitly examined, the same results stated above are valid beyond it. 


\section{The Structure of the Zero-Mode Constraint}

We reexamine the zero-mode constraint for the NG boson with the mass term. The crucial feature of the NG phase is the singular behavior of the zero mode (3.7) which is explicitly confirmed by the lowest order solution of the linear sigma model. The purpose of this Appendix is to confirm (3.7) beyond the leading approximation. For simplicity, consider the bosonic sector of the linear sigma model in (4.15) and (4.16). By redefining the lowest order solution

$$
\begin{aligned}
\omega_{\pi}^{(1)} & =\frac{\lambda}{2 L} \int_{-L}^{L} d x^{-}\left(-m_{\pi}^{2}+\partial_{\perp}^{2}\right)^{-1}\left(\varphi_{\pi}^{3}+\varphi_{\pi} \varphi_{\sigma}^{2}+2 v \varphi_{\pi} \varphi_{\sigma}\right) \\
& \equiv \mathcal{D}_{\pi} \Phi_{\pi}\left(x^{+}, x^{\perp}\right)
\end{aligned}
$$

and

$$
\begin{aligned}
\omega_{\sigma}^{(1)} & =\frac{\lambda}{2 L} \int_{-L}^{L} d x^{-}\left(-m_{\sigma}^{2}+\partial_{\perp}^{2}\right)^{-1}\left(\varphi_{\sigma}^{3}+\varphi_{\sigma} \varphi_{\pi}^{2}+v \varphi_{\pi}^{2}+3 v \varphi_{\sigma}^{2}\right) \\
& \equiv \mathcal{D}_{\sigma} \Phi_{\sigma}\left(x^{+}, x^{\perp}\right)
\end{aligned}
$$

where $\mathcal{D}_{\pi} \equiv\left(-m_{\pi}^{2}+\partial_{\perp}^{2}\right)^{-1}$ and $\mathcal{D}_{\sigma} \equiv\left(-m_{\sigma}^{2}+\partial_{\perp}^{2}\right)^{-1}$, the second order perturbative solution of the NG-boson zero mode is given by

$$
\begin{aligned}
\omega_{\pi}^{(2)} & =\frac{\lambda}{2 L} \int_{-L}^{L} d x^{-} \mathcal{D}_{\pi}\left\{\frac{1}{2}\left[\left(\mathcal{D}_{\sigma} \Phi_{\sigma}\right) \varphi_{\sigma} \varphi_{\pi}+\varphi_{\sigma}\left(\mathcal{D}_{\sigma} \Phi_{\sigma}\right) \varphi_{\pi}+\varphi_{\pi}\left(\mathcal{D}_{\sigma} \Phi_{\sigma}\right) \varphi_{\sigma}+\varphi_{\sigma} \varphi_{\pi}\left(\mathcal{D}_{\sigma} \Phi_{\sigma}\right)\right]\right. \\
& \left.+\left[\left(\mathcal{D}_{\pi} \Phi_{\pi}\right) \varphi_{\pi}^{2}+\varphi_{\pi}^{2}\left(\mathcal{D}_{\pi} \Phi_{\pi}\right)+\varphi_{\pi}\left(\mathcal{D}_{\pi} \Phi_{\pi}\right) \varphi_{\pi}\right]+\frac{1}{2}\left[\left(\mathcal{D}_{\pi} \Phi_{\pi}\right) \varphi_{\sigma}^{2}+\varphi_{\sigma}^{2}\left(\mathcal{D}_{\pi} \Phi_{\pi}\right)\right]\right\} \quad, \quad(\mathrm{D} .10)
\end{aligned}
$$

which leads to

$$
\begin{aligned}
\int d^{3} \vec{x} \omega_{\pi}^{(2)} & \sim-\frac{\lambda}{m_{\pi}^{2}} \int d^{3} \vec{x}\left\{\frac{1}{2}\left[\left(\mathcal{D}_{\sigma} \Phi_{\sigma}\right) \varphi_{\sigma} \varphi_{\pi}+\varphi_{\sigma}\left(\mathcal{D}_{\sigma} \Phi_{\sigma}\right) \varphi_{\pi}+\varphi_{\pi}\left(\mathcal{D}_{\sigma} \Phi_{\sigma}\right) \varphi_{\sigma}+\varphi_{\sigma} \varphi_{\pi}\left(\mathcal{D}_{\sigma} \Phi_{\sigma}\right)\right]\right. \\
& +\left[\left(\partial_{\perp}^{-2} \Phi_{\pi}\right) \varphi_{\pi}^{2}+\varphi_{\pi}^{2}\left(\partial_{\perp}^{-2} \Phi_{\pi}\right)+\varphi_{\pi}\left(\partial_{\perp}^{-2} \Phi_{\pi}\right) \varphi_{\pi}\right] \\
& \left.+\frac{1}{2}\left[\left(\partial_{\perp}^{-2} \Phi_{\pi}\right) \varphi_{\sigma}^{2}+\varphi_{\sigma}^{2}\left(\partial_{\perp}^{-2} \Phi_{\pi}\right)\right]\right\} \\
& \sim \frac{1}{m_{\pi}^{2}}
\end{aligned}
$$


in the $m_{\pi} \rightarrow 0$ limit. Repeating the similar procedure, such singular behaviors of the zero mode for higher order perturbative solutions $(n>1)$,

$$
\int d^{3} \vec{x} \omega_{\pi}^{(n)} \sim \frac{1}{m_{\pi}^{2}}
$$

can also be confirmed inductively.

Here it may be interesting to consider the same problem in two-dimensions where a continuous symmetry cannot be broken spontaneously due to Coleman's theorem [16]. In two dimensions, the leading order solution of the zero modes is given by

$$
\begin{aligned}
& \omega_{\pi}^{(1)}=-\frac{1}{m_{\pi}^{2}} \Phi_{\pi}, \\
& \omega_{\sigma}^{(1)}=-\frac{1}{m_{\sigma}^{2}} \Phi_{\sigma},
\end{aligned}
$$

where $\Phi_{\pi, \sigma}$ depend on $x^{+}$only. Without integral in $x^{\perp}, \omega_{\pi}^{(1)}$ by itself becomes divergent as $m_{\pi} \rightarrow 0$ in sharp contrast to the four-dimensional case. The peculiarity of two dimensions becomes clearer in the higher order solution. In fact, up to the operator ordering, the second order solution

$$
\omega_{\pi}^{(2)}=\frac{\lambda}{2 L} \int_{-L}^{L} d x^{-} \frac{1}{m_{\pi}^{2}}\left\{\frac{1}{m_{\pi}^{2}} \Phi_{\pi} \varphi_{\sigma}^{2}+\frac{2}{m_{\sigma}^{2}} \Phi_{\sigma}+\frac{3}{m_{\pi}^{2}} \Phi_{\pi} \varphi_{\pi}^{2}\right\},
$$

leads to

$$
\omega_{\pi}^{(2)} \sim \frac{1}{m_{\pi}^{4}} \quad\left(m_{\pi} \sim 0\right) .
$$

In general the $n$-th order perturbative solution of the NG-boson zero mode behaves as

$$
\omega_{\pi}^{(n)} \sim \frac{1}{m_{\pi}^{2 n}} .
$$

The singular behavior becomes worse in higher orders. In other words, the NG-boson field in two dimensions is ill-defined due to its wrong "infrared behavior" at $p^{+} \rightarrow 0$. In this sense the NG phase cannot be realized in two dimensions in conformity with Coleman's theorem [16], even if we apply our method by introducing the NG-boson mass as a regulator. 


\section{References}

[1] P.A.M. Dirac, Rev. Mod. Phys. 21, 392 (1949).

[2] See, for example, S.J. Brodsky, G. McCartor, H.C. Pauli, and S.S. Pinsky, Particle World 3, 109 (1993) and references therein.

[3] K.G. Wilson, T.S. Walhout, A. Harindranath, W. Zhang, R. J. Perry, and S.D. Glazek, Phys. Rev. D49, 6720 (1994)

[4] H. Leutwyler, J.R. Klauder, and L. Streit, Nuovo Cim. 66A, 536 (1970).

[5] T. Maskawa and K. Yamawaki, Prog. Theor. Phys. 56, 270 (1976).

[6] A. Casher, Phys. Rev. D14, 452 (1976).

[7] H.C. Pauli and S.J. Brodsky, Phys. Rev. D32, 1993 (1985); ibid 2001 (1985).

[8] A. Harindranath and J.P. Vary, Phys. Rev. D36, 1141 (1987); ibid D37, 1064 (1988).

[9] T. Eller, H.C. Pauli, and S.J. Brodsky, Phys. Rev. D35, 1493 (1987); T. Eller, H.C. Pauli, Z. Phys. C42, 59 (1989).

[10] M. Burkardt, Nucl. Phys. A504, 762 (1989); K. Hornbostel, S.J. Brodsky, and H.C. Pauli, Phys. Rev. D41, 3814 (1990).

[11] A.C. Tang, S.J. Brodsky, and H.C. Pauli, Phys. Rev. D44, 1842 (1991); M. Kaluza and H.C. Pauli, Phys. Rev. D45, 2968 (1992); L.C.L. Hollenberg, K. Higashijima, R.C. Warner, and B.H.J. McKellar, Prog. Theor. Phys. 87, 3411 (1991); M. Krautgärtner, H.C. Pauli, and F. Wölz, Phys. Rev. D45, 3755 (1992); H.C. Pauli, hep-th/9608035. U. Trittmann and H.C. Pauli, hep-th/9704215 U. Trittmann and H.C. Pauli, hepth/9705021 
[12] T. Heinzl, S. Krusche, S. Simbürger, and E. Werner, Z. Phys. C 56, 415 (1992).

[13] D.G. Robertson, Phys. Rev. D47, 2549 (1993).

[14] C.M. Bender, S. Pinsky, and B. Van de Sande, Phys. Rev. D48, 816 (1993); S. Pinsky and B. Van de Sande, Phys. Rev. D49, 2001 (1994); S. Pinsky, B. Van de Sande, and J. Hiller, Phys. Rev. D51, 726 (1995).

[15] Y. Kim, S. Tsujimaru, and K. Yamawaki, Phys. Rev. Lett 74, 4771 (1995).

[16] S. Coleman, Commun. Math. Phys. B1, 259 (1973).

[17] N. Nakanishi and K. Yamawaki, Nucl. Phys. B122, 15 (1977).

[18] P. Steinhardt, Ann. Phys. 128, 425 (1980).

[19] T. Heinzl, S. Krushe, and E. Werner, Phys. Lett. B277, 54 (1991).

[20] G. McCartor and D.G. Robertson, Z. Phys. C53, 679 (1992).

[21] C.M. Bender, L. R. Mead, and S. S. Pinsky, Phys. Rev. Lett. 56, 2445 (1986).

[22] S. Weinberg, Phys. Rev. 177, 2604 (1969); Phys. Rev. Lett. 65, 1177 (1990).

[23] M. Bando, T. Kugo, and K. Yamawaki, Phys. Rep. 164, 217 (1988).

[24] S.L. Adler, Phys. Rev. 140, B736 (1965); W.I. Weisberger, Phys. Rev. 143, 1302 (1966).

[25] F. Gilman and H. Harari, Phys. Rev. 165, 1803 (1968).

[26] M. Ida, Prog. Theor. Phys. 51, 1521 (1974); M. Ida, T. Tajima, and K. Yamawaki, Prog. Theor. Phys. 52, 1583 (1974).

[27] Y. Nambu and G. Jona-Lasinio, Phys. Rev. 112345 (1961).

[28] R. Carlitz, D. Heckathorn, J. Kaur, and W.K. Tung, Phys. Rev. D11, 1234 (1975). 
[29] N. Nakanishi and H. Yabuki, Lett. Math. Phys. 1371 (1977).

[30] E.V. Prokhvatilov and V.A. Franke, Sov. J. Nucl. Phys. 49, 688 (1989); F. Lenz, M. Thies, K. Yazaki, S. Levit, Ann. Phys. 208, 1 (1991); K. Hornbostel, Phys. Rev. D45 3781 (1992).

[31] E.V. Prokhvatilov, H.L. Naus, and H.J. Pirner, Phys. Rev. D51, 2933 (1995); M. Burkardt, Adv. Nucl. Phys.23, 1 (1996); J.P. Vary, T.J. Fields, H.-J. Pirner, Phys. Rev. D53, 7231 (1996); H.L. Naus, H.J. Pirner, T.J. Fields, J.P. Vary, hep-th/9704135.

[32] S.-J. Chang and S.-K. Ma, Phys. Rev. 180, 1506 (1969).

[33] D. Mustaki, Phys. Rev. D 38, 1260 (1988) 